\title{
ON A DETERMINANTAL FORMULA OF TADIĆ
}

\author{
EREZ LAPID AND ALBERTO MÍNGUEZ
}

\begin{abstract}
We study a special class of irreducible representations of $\mathrm{GL}_{n}$ over a local non-Archimedean field which we call ladder representations. This is a natural class in the admissible dual which contains the Speh representations. We show that the Tadic determinantal formula is valid for this class and analyze the standard modules pertaining to these representations.
\end{abstract}

\section{INTRODUCTION}

Let $F$ be a non-Archimedean locally compact field and, for every integer $n \geq 0$, set $G_{n}=\mathrm{GL}_{n}(F)$. (with the convention that $G_{0}$ is the trivial group). Denote by $\mathscr{R}_{n}$ the Grothendieck group of the category of smooth representations of $G_{n}$ of finite length, and let $\mathscr{R}=\oplus_{n \geq 0} \mathscr{R}_{n}$ with the product structure defined by normalized parabolic induction. The commutative ring $\mathscr{R}$ (with the one-dimensional representation of $G_{0}$ as the identity element) was introduced by Zelevinsky, who showed that it is freely generated by the essentially square-integrable representations (of any $G_{n}$ ) [Zel80]. Hence, the monomials in these generators (the so-called standard modules) form a basis for $\mathscr{R}$. Another natural basis for $\mathscr{R}$ is given by the irreducible representations. The change of basis matrix is unitriangular (in an appropriate sense) and the coefficients can be expressed in terms of the Kazhdan-Lusztig polynomials. This fact was conjectured by Zelevinsky [Zel81] (and in a more precise form in [Zel85]) and proved in [CG97]. While in principle this solves the problem of decomposing a standard module into irreducible constituents, in practice the coefficients are very complicated. Nevertheless, in the case of a Speh representation, Tadić obtained a remarkable formula expressing its character as a linear combination of characters of standard modules with coefficients \pm 1 [Tad95]. Tadić's ingenious argument transfers the problem to a question about complex groups. Later on, the argument was simplified by Chenevier and Renard [CR08] by a clever use of the Desnanot-Jacobi identity of determinants (also known as Dodgson's rule of determinants). Both proofs rely on the determination of the composition series at the edge of a complementary series, and thus they heavily rely on unitarity.

Our purpose in this note is to provide a different proof of Tadić's formula which yields additional information on the structure of the Langlands quotient, and at the same time extends to a wide class of representations which we call ladder representations. (We caution

Date: March 7, 2012.

First named author partially supported by a grant from the Israel Science Foundation.

Second named author partially supported by ANR-10-BLANC 0114, EPSRC grant EP/G001480/1, MTM2010-19298 and FEDER. 
that in the literature there is an unrelated, older notion of ladder representations for unitary groups.) The extra piece of information (part 1 of Theorem 1 below) is used in [FLO] to prove for this class of representations a conjecture of Jacquet about the existence of functionals invariant under a unitary group.

Except for the Speh representations, ladder representations are not unitary. Instead of unitarity, we use Jacquet module technique. Unlike in other scenarios, it is necessary to study not only the semisimplification of the Jacquet module, but the finer structure of its submodules.

Curiously, using our result one can turn the table and use the Desnanot-Jacobi determinantal identity to obtain the decomposition of certain induced spaces (including the ends of complementary series) as a corollary.

To state our results, let us introduce some more notation. Denote by $\nu$ the character |det| on any $G_{n}$. (The $n$ will be implicit and hopefully clear from the context.) For any smooth representation $\pi$ of $G_{n}$ and $a \in \mathbb{R}$ denote by $\pi \nu^{a}$ the representation obtained from $\pi$ by twisting it by the character $\nu^{a}$. If $\pi_{1}, \ldots, \pi_{r}$ are smooth representations of $G_{n_{1}}, \ldots, G_{n_{r}}$ respectively, we will denote as usual by

$$
\pi_{1} \times \pi_{2} \times \cdots \times \pi_{r}
$$

the representation of $G_{n_{1}+n_{2}+\cdots+n_{r}}$ parabolically induced (normalized induction) from the representation $\pi_{1} \otimes \pi_{2} \otimes \cdots \otimes \pi_{r}$ of the standard parabolic subgroup of $G_{n_{1}+n_{2}+\cdots+n_{r}}$ of type $\left(n_{1}, \ldots, n_{r}\right)$ (with Levi subgroup $G_{n_{1}} \times \cdots \times G_{n_{r}}$ ).

Throughout we fix a positive integer $d$ and an irreducible cuspidal (not necessarily unitary) representation $\sigma$ of $G_{d}$. By a segment $[a, b]$ we mean a set of elements $\{a, a+1, \ldots, b\}$ where $b \geq a$ are integers. For any segment $[a, b]$ we denote by $\Delta([a, b])$ the unique irreducible quotient of $\sigma \nu^{a} \times \cdots \times \sigma \nu^{b}$. It is an essentially square-integrable representation of $\mathrm{GL}_{d(b-a+1)}$. The map $[a, b] \mapsto \Delta([a, b])$ is a bijection between the segments and the essentially square-integrable representations whose cuspidal support is contained $\left\{\sigma \nu^{i}: i \in \mathbb{Z}\right\}$ $\left[\right.$ Zel80, §9.3]. We also write $\Delta([a, a-1])=1$ (the one-dimensional representation of $\left.\mathrm{GL}_{0}\right)$ for any $a \in \mathbb{Z}$ and $\Delta([a, b])=0$ if $b<a-1$.

Let $\left[a_{1}, b_{1}\right], \ldots,\left[a_{t}, b_{t}\right]$ be segments and let $\Delta_{i}=\Delta\left(\left[a_{i}, b_{i}\right]\right)$. Assume that $\Delta_{i}$ does not precede $\Delta_{j}$ for any $i<j$, i.e., we do not have $a_{i}<a_{j} \leq b_{i}+1<b_{j}+1$ for $i<j$. Then the representation $\Delta_{1} \times \cdots \times \Delta_{t}$ admits a unique irreducible quotient, the Langlands quotient, which we denote by $\mathrm{L}\left(\Delta_{1}, \ldots, \Delta_{t}\right)$ (see for example [Rod82, Théorème 3$]$ ).

Assume now that $a_{1}>\cdots>a_{t}$ and $b_{1}>\cdots>b_{t}$. In this case we say that $\Delta_{1}, \ldots, \Delta_{t}$ is a ladder and call $\mathrm{L}\left(\Delta_{1}, \ldots, \Delta_{t}\right)$ a ladder representation. A particularly important subclass is the Speh representations where for every $i=1, \ldots, t-1, a_{i+1}=a_{i}-1$ and $b_{i+1}=b_{i}-1$. These representations comprise the building blocks for the unitary dual of $G_{n}([\operatorname{Tad} 86])$.

Let $S_{t}$ be the symmetric group on $\{1, \ldots, t\}$. For any $w \in S_{t}$ let $\mathcal{I}_{w}=\Delta\left(\left[a_{w(1)}, b_{1}\right]\right) \times$ $\cdots \times \Delta\left(\left[a_{w(t)}, b_{t}\right]\right)$. (This is 0 unless $a_{w(i)} \leq b_{i}+1$ for all $i$.) In particular, for $k=1, \ldots, t-1$ let $\mathscr{K}_{k}=\mathcal{I}_{s_{k}} \subseteq \mathcal{I}_{\text {Id }}$ where $s_{k}$ is the transposition $(k, k+1)$.

Theorem 1. Assume that $\Delta_{1}, \ldots, \Delta_{t}$ is a ladder and let $\pi=\mathrm{L}\left(\Delta_{1}, \ldots, \Delta_{t}\right)$. Then

(i) The maximal proper submodule of $\Delta_{1} \times \cdots \times \Delta_{t}$ is $\sum_{k=1}^{t-1} \mathscr{K}_{k}$. 
(ii) $\pi$ satisfies Tadić's determinantal formula

$$
\pi=\sum_{w \in S_{t}} \operatorname{sgn} w \mathcal{I}_{w}
$$

in $\mathscr{R}$.

As mentioned before, the formula (1.1) was proved by Tadic in the case of Speh representations [Tad95] and his proof was simplified in [CR08].

In the course of proving Theorem 1 we will analyze the Jacquet functor $J(\pi)$ of $\pi$ with respect to the parabolic subgroup of type $(d, \ldots, d)$ and obtain a formula for its character. Remarkably, $J(\pi)$ is the quotient of $J\left(\Delta_{1} \times \cdots \times \Delta_{t}\right)$ by the sum of generalized eigenspaces (with respect to the torus action). We will introduce a certain directed graph $\mathscr{E}(\pi)$ whose vertex set consists of the segments $\Delta_{1}, \ldots, \Delta_{t}$ drawn sequentially in the plane, and the edges are horizontal and diagonal arrows. The character of $J(\pi)$ will be expressed in terms of the possible vertex labellings of $\mathscr{E}(\pi)$ which are increasing with respect to the arrows. See Theorem 7 for the precise statement. In particular, the length of $J(\pi)$ is equal, in the terminology of [Sta99, $\S 7.10]$, to the number of standard Young Tableaux of the skew Young diagram $\left(a_{1}+1, \ldots, a_{t}+t\right) /\left(b_{1}+1, \ldots, b_{t}+t\right)$. Incidently, this can be computed by a well-known determinantal formula which is a consequence of the Jacobi-Trudi identity (cf. [Sta99, Corollary 7.16.3]). We may regard (1.1) as a $p$-adic analogue of the Jacobi-Trudi identity.

We remark that for general irreducible representations of $\mathrm{GL}_{n}$ there is no known or conjectural simple description of their Jacquet modules in terms of the Langlands (or Zelevinsky) data.

Theorem 1 has an interesting application which was pointed out to us by Marko Tadić and which is incorporated here with his kind permission. Namely, one can compute the full derivative (in the sense of Bernstein-Zelevinsky) of a ladder representation in terms of subordinate (in the sense of Zelevinsky) ladder representations. This expression had been conjectured by Tadić some time ago in the case of Speh representations [Tad87]. Interestingly enough, already in this case one has to use non-unitary ladder representations.

In the last section we make some further comments about ladder representations and beyond. We first conjecture that roughly speaking, in the ladder case, the decomposition of $\Delta_{1} \times \cdots \times \Delta_{t}$ is quite uniform and does not depend in an essential way on the segments (except that some constituents may disappear, depending on the ordering of $\left.a_{1}, b_{1}, \ldots, a_{t}, b_{t}\right)$. Our second conjecture is a generalization of Theorem 1 part 1 to an arbitrary irreducible representation. This conjecture seems to be the first one addressing the fine structure of the Langlands quotient in the corresponding standard module. (Incidently, this conjecture, as well as the class of ladder representations, was inspired by analyzing functionals invariant under unitary groups - cf. [FLO].) We also provide a simple example of non-ladder irreducible representations which are not (fully) parabolically induced from representations of smaller $\mathrm{GL}_{n}$ 's.

To summarize, it seems that many results about Speh representations can be extended to ladder representations. Thus, the latter provide a suitable algebraic envelope for the 
former which avoids unitarity. We view the family of ladder representations as broad enough to include many interesting representations on the one hand and on the other hand sufficiently restricted so that one can approach their structure. Thus it provides a "litmus paper" for testing conjectures about representations of $G_{n}$ (for instance Conjecture 2 alluded to above). See [BLM] for a follow-up about irreducibility questions.

Finally, we remark that all the results and the proofs in this paper except for $\S 5.5$ carry over to inner forms of $\mathrm{GL}_{n}$ with minor changes.

Acknowledgement. The authors would like to thank Shaun Stevens and the University of East Anglia for their hospitality. Also, the second named author wishes to thank the Hebrew University of Jerusalem for its hospitality. We also thank Ioan Badulescu and Laurent Clozel for useful discussions and Marko Tadić for his contribution in §5.5. Finally, we thank Ira Gessel for correspondence related to Proposition 10.

\section{NotATION AND PRELIMINARIES}

2.1. Throughout this article, we fix a non-Archimedean locally compact field $F$. Let $G$ be the group of $F$-points of a connected reductive group defined over $F$, with the usual topology. We will only consider smooth representations of $G$, that is, representations such that the stabilizer of every vector is an open subgroup of $G$.

Fix a minimal $F$-parabolic subgroup $P_{0}$ of $G$ and let $M_{0}$ be a Levi factor of $P_{0}$ defined over $F$. We denote by $W_{G}$ the spherical Weyl group, defined by

$$
W_{G}=\mathrm{N}_{G}\left(M_{0}\right) / M_{0},
$$

where $\mathrm{N}_{G}\left(M_{0}\right)$ is the normalizer of $M_{0}$ in $G$. A parabolic subgroup $P$ of $G$ will be called standard if it contains $P_{0}$. Henceforth, the letter $P$ will always denote a standard parabolic subgroup of $G$ with an implicit standard Levi decomposition $P=M U$.

Let $(\tau, \mathrm{V})$ be a representation of $M$, regarded as a representation of $P$ on which $U$ acts trivially. We denote by $I_{M}(\tau)=I_{M}^{G}(\tau)=\operatorname{Ind}_{P}^{G} \tau$, the representation of $G$ induced from $\tau$. (We will always mean normalized induction.) We view $I_{M}^{G}$ as a functor. Its left adjoint, the Jacquet functor with respect to $P$, will be denoted by $J_{M}$. For any representation $\pi$ of $G$, we let $j_{M}=j_{M, \pi}: \pi \rightarrow J_{M}(\pi)$ be the canonical projection.

An irreducible representation $\pi$ of $G$ is called cuspidal if it is not a composition factor of any representation of the form $I_{M}^{G}(\tau)$ with $P$ a proper parabolic subgroup of $G$ and $\tau$ a representation of $M$.

We denote by $\operatorname{Irr} G$ (resp. $\operatorname{Irr}_{c} G$ ) the set of equivalence classes of (resp. cuspidal) irreducible representations of $G$. For any $\pi \in \operatorname{Irr} G$ there exists, up to conjugacy, a unique pair $(M, \rho)$ consisting of a Levi subgroup $M$ of $G$ and $\rho \in \operatorname{Irr}_{c} M$ such that $\pi$ is a composition factor of $I_{M}^{G}(\rho)$. We call it the cuspidal support of $\pi$ and write it $\operatorname{supp}(\pi)$.

Let $\mathscr{R}(G)$ denote the Grothendieck group of the category of smooth representations of $G$ of finite length. The image of a representation $\pi$ of $G$ finite length in $\mathscr{R}(G)$ will be denoted by $[\pi]$. The Jacquet functor $J_{M}$ induces a homomorphism $\mathbf{J}_{M}: \mathscr{R}(G) \rightarrow \mathscr{R}(M)$. Thus $\mathbf{J}_{M}(\pi)$ is the semisimplification $\left[J_{M}(\pi)\right]$ of $J_{M}(\pi)$. 
2.2. For any integer $n \geq 0$, set $G_{n}=\mathrm{GL}_{n}(F)$. Let $P_{0}$ be the Borel subgroup of upper triangular matrices and let $U_{0}$ be its unipotent radical consisting of upper unitriangular matrices. The standard parabolic subgroups of $G$ are in bijection with compositions $n=$ $n_{1}+\cdots+n_{t}$. The corresponding standard Levi subgroup is the group of block diagonal invertible matrices with block sizes $n_{1}, \ldots, n_{t}$. It is isomorphic to $G_{n_{1}} \times \cdots \times G_{n_{r}}$.

As in the introduction, if $\rho_{1}, \ldots, \rho_{r}$ are representations of $G_{n_{1}}, \ldots, G_{n_{r}}$ respectively, we will denote by

$$
\rho_{1} \times \rho_{2} \times \cdots \times \rho_{r}=I_{M}^{G_{n}}(\rho)
$$

the corresponding induced representation where $\rho$ is the representation $\rho_{1} \otimes \rho_{2} \otimes \cdots \otimes \rho_{r}$ of $M$. Here $P=M U$ is the standard parabolic subgroup corresponding to the composition $n=n_{1}+\cdots+n_{r}$.

Given $\pi \in \operatorname{Irr} G_{n}$, we can view the cuspidal support of $\pi$ as the unique multi-set (i.e., set with multiplicities) $\left(\rho_{1}, \ldots, \rho_{r}\right), \rho_{i} \in \operatorname{Irr}_{c} G_{n_{i}}, n=n_{1}+\cdots+n_{r}$ such that $\pi$ is a composition factor of $\rho_{1} \times \rho_{2} \times \cdots \times \rho_{r}$.

2.3. Let $\mathscr{R}_{n}=\mathscr{R}\left(G_{n}\right)$ and $\mathscr{R}=\oplus_{n \geq 0} \mathscr{R}_{n}$. Then $\mathscr{R}$ is a graded commutative ring with the product defined by (2.1). The identity element is the one-dimensional representation of $G_{0}$. The natural ordering on $\mathscr{R}$ will be denoted by $\leq$. With respect to the addition $\mathscr{R}$ forms a lattice ordered group. We say that two non-negative elements of $\mathscr{R}$ are disjoint if their meet is 0 .

We also write $\operatorname{Irr}=\coprod_{n \geq 0} \operatorname{Irr} G_{n}$.

2.4. Throughout the article, we fix a positive integer $d$ and $\sigma \in \operatorname{Irr}_{c} G_{d}$ (not necessarily unitary). Write $n=m d$. Let $P_{\mathfrak{P}}=M_{\mathfrak{P}} U_{\mathfrak{P}}$ be the parabolic subgroup of type $(d, \ldots, d)$ in $G_{n}$ with $M_{\mathfrak{P}} \simeq G_{d} \times \cdots \times G_{d}$. For simplicity we write $J=J_{\mathfrak{P}}, j=j_{\mathfrak{P}}$ and $\mathbf{J}=\mathbf{J}_{\mathfrak{P}}$ : $\mathscr{R}\left(G_{n}\right) \rightarrow \mathscr{R}\left(G_{d} \times \cdots \times G_{d}\right)=\mathscr{R}\left(G_{d}\right) \otimes \cdots \otimes \mathscr{R}\left(G_{d}\right)$ ( $m$ times). Henceforth, we will only consider standard parabolic and Levi subgroups containing $M_{\mathfrak{P}}$. Note that then $M$ is of type $n_{1}, \ldots, n_{t}$ where $d$ divides all $n_{i}$ 's. We write $n_{i}=d m_{i}$.

2.5. Denote by $\mathbb{Z}_{\sigma}$ the set of cuspidal representations of the form $\sigma \nu^{i}$, for $i \in \mathbb{Z}$. We denote by $\operatorname{Irr}_{\sigma} \subseteq$ Irr the set of equivalence classes of irreducible representations of $G_{n}$ (any $n$ ) whose cuspidal support is contained in $\mathbb{Z}_{\sigma}$. We denote by $\mathscr{C}=\mathscr{C}_{\sigma}^{G_{n}}$ the category of the finite length representations of $G_{n}$ with the property that all their irreducible subquotients belong to $\operatorname{Irr}_{\sigma}$. More generally, for any standard Levi $M$ (containing $M_{\mathfrak{P}}$ ) let $\mathscr{C}^{M}=\mathscr{C}_{\sigma}^{M}$ be the category of finite length representations (of $M$ ) with a similar property. We continue to write $J$ (or $J^{M}$ ) for the Jacquet functor with respect to $P_{\mathfrak{P}} \cap M$.

2.6. Let $W$ be the Weyl group of $G_{n}$, identified with $S_{n}$ by

$$
\begin{aligned}
S_{n} & \rightarrow W \\
w & \mapsto\left(\delta_{i, w(j)}\right)
\end{aligned}
$$

and let $W^{M}$ be the Weyl group of $M$. We identify $W^{M} \backslash W$ with the set $\tilde{\Omega}_{M}$ of left- $W^{M_{-}}$ reduced elements of $W$, i.e. the elements of $W$ of minimal length in their left $W^{M}$-coset. 
Of particular importance will be the subset $\Omega_{M}$ of $\tilde{\Omega}_{M}$ defined as follows. Let $W_{0}$ be the Weyl group of $G_{d} \times \cdots \times G_{d}$ and let $W^{0}$ be the set of reduced elements in $W / W_{0}$ which normalize $M_{\mathfrak{P}}$. Note that $W^{0}$ is a subgroup of $W$ which we identify with $S_{m}$. We define $\Omega_{M}=\left(W^{0} \cap W^{M}\right) \backslash W^{0}$ identified with a subset of $\tilde{\Omega}_{M}$, i.e., $\Omega_{M}$ is the set of elements of $W^{0}$ which are of minimal length in their left $W^{M}$-coset. Explicitly,

$$
\Omega_{M}=\left\{w \in S_{m}: w^{-1}(i)<w^{-1}(i+1) \forall i \neq m_{1}, m_{1}+m_{2}, \ldots, m_{1}+\cdots+m_{t-1}\right\} .
$$

In particular, $\left|\Omega_{M}\right|=\left(\begin{array}{c}m \\ m_{1} m_{2} \ldots m_{t}\end{array}\right)$.

We note that for any $w \in \Omega_{M}$ we have

$$
w U_{\mathfrak{P}} w^{-1} \cap M=U_{\mathfrak{P}} \cap M
$$

and

$$
w U_{\mathfrak{P}} w^{-1} \cap P=\left(U_{\mathfrak{P}} \cap M\right)\left(w U_{\mathfrak{P}} w^{-1} \cap U\right) .
$$

Remark 1. $\Omega_{M}$ is the set denoted by $W^{M, M_{\mathfrak{P}}}$ in $[\mathrm{BZ77}, \S 2.11]$. Note that $\Omega_{M}$ normalizes $M_{\mathfrak{P}}$.

Recall the Bruhat decomposition $G=\cup_{w \in W} P_{0} w U_{0}$ and the relative Bruhat decomposition

$$
G=\cup_{w \in \tilde{\Omega}_{M}} P w U_{0}
$$

We denote the Bruhat order on $W$ by $\leq$. Recall that $w_{1} \leq w_{2}$ if and only if $P_{0} w_{1} U_{0} \subseteq$ $\overline{P_{0} w_{2} U_{0}}$ in the $p$-adic topology of $G_{n}$. We refer to [BB05] for standard facts about the Bruhat order. The Bruhat order induces a partial order on $\tilde{\Omega}_{M}$ and on $\Omega_{M}$. Once again, for $w_{1}, w_{2} \in \tilde{\Omega}_{M}$ we have $w_{1} \leq w_{2}$ if and only if $P w_{1} U_{0} \subseteq \overline{P w_{2} U_{0}}$.

The Bruhat order gives rise to a topology on $W$. Namely, $A \subseteq W$ is open if and only if whenever $w, w^{\prime} \in W$ and $w \geq w^{\prime} \in A$, we have $w \in A$. Equivalently, a subset $A \subseteq W$ is open if and only if $P_{0} A U_{0}$ is open in $G_{n}$. For instance, for any $w \in W$ the subsets $W_{\geq w}=\left\{w^{\prime} \in W: w^{\prime} \geq w\right\}$ and $W_{>w}=\left\{w^{\prime} \in W: w^{\prime}>w\right\}$ are open. Similarly for $\tilde{\Omega}_{M, \geq w}$ in $\tilde{\Omega}_{M}$, etc.. (We topologize subsets of $W$ by the relative topology.) Note that $W_{\geq w}=W^{M} \tilde{\Omega}_{M, \geq w}$ for any $w \in \tilde{\Omega}_{M}$.

2.7. Let $\tau \in \mathscr{C}^{M}$ and let $\Pi=I_{M}(\tau)$. For any open subset $\omega$ in $\tilde{\Omega}_{M}$ consider the $P_{\mathfrak{P}^{-}}$ invariant subspace

$$
\Pi_{\omega}=\Pi_{M, \omega}:=\left\{\varphi \in I_{M}(\tau): \operatorname{supp}(\varphi) \subseteq P \omega U_{0}\right\}=\left\{\varphi \in I_{M}(\tau):\left.\varphi\right|_{G_{n} \backslash P \omega U_{0}} \equiv 0\right\} .
$$

Let $J(\Pi)_{M, \omega}$ be its image under $j$, which is a subrepresentation of $J(\Pi)$. (We often omit $M$ if it's clear from the context.) In particular for any $w \in \tilde{\Omega}_{M}$, we can consider $\Pi_{\geq w}$, $\Pi_{>w}, J(\Pi)_{\geq w}$ and $J(\Pi)_{>w}$. By [BZ77, §2.12], fixing a choice of Haar measures, the map

$$
\tilde{p}_{w}: \varphi \mapsto \int_{\left(U_{\mathfrak{P}} \cap w^{-1} U_{\mathfrak{P}} w\right) \backslash U_{\mathfrak{P}}} j_{\tau}(\varphi(w u)) d u \in J(\tau), \quad \varphi \in \Pi_{\geq w}
$$


induces a surjective homomorphism

$$
p_{M, \tau, w}=p_{w}: J(\Pi)_{\geq w} \rightarrow \begin{cases}J(\tau)^{w} & \text { if } w \in \Omega_{M}, \\ 0 & \text { otherwise }\end{cases}
$$

whose kernel is $J(\Pi)_{>w}$. (Note that $\tilde{p}_{w}$ is well-defined because $P w U_{0}$ is closed in $P W_{\geq w} U_{0}$.) Here $J(\tau)^{w}$ is the vector space $J(\tau)$ with the twisted action of $M_{\mathfrak{P}}$ by $w$.

Note that if $\beta \subseteq \tau$ then $J\left(I_{M}(\beta)\right) \subseteq J\left(I_{M}(\tau)\right)$ and $p_{M, \beta, w}$ is the restriction of $p_{M, \tau, w}$.

2.8. Now let $L$ be a standard Levi subgroup of $M$. Set $\tilde{\Omega}_{L}^{M}=\tilde{\Omega}_{L} \cap W^{M}$ and $\Omega_{L}^{M}=$ $\Omega_{L} \cap W^{M}$. Recall that the map $\left(w_{1}, w_{2}\right) \mapsto w_{1} w_{2}$ defines bijections $\tilde{\Omega}_{L}^{M} \times \tilde{\Omega}_{M} \rightarrow \tilde{\Omega}_{L}$ and $\Omega_{L}^{M} \times \Omega_{M} \rightarrow \Omega_{L}$. Let $\varrho \in \mathscr{C}^{L}$ and $\tau=I_{L}^{M}(\varrho)$, so that $\Pi=I_{M}(\tau) \simeq \tilde{\Pi}:=I_{L}(\varrho)$. Let $\iota_{L}^{M}$ the equivalence of representations $\iota_{L}^{M}: \tilde{\Pi} \rightarrow \Pi$ defined by $\iota_{L}^{M} \varphi(g)=(h \mapsto \varphi(h g))$. Correspondingly we have $J\left(\iota_{L}^{M}\right): J(\tilde{\Pi}) \rightarrow J(\Pi)$. For any $w \in \Omega_{L}^{M}$ we may consider $\tau_{\geq w}$ and $J(\tau)_{L, \geq w}$. The following result is probably well known. For convenience we include a proof.

Proposition 2 (Compatibility with induction in stages). Under the above assumptions suppose that $w=w_{1} w_{2} \in \Omega_{L}$ where $w_{1} \in \Omega_{L}^{M}, w_{2} \in \Omega_{M}$. Then

$$
\begin{gathered}
\iota_{L}^{M}\left(\tilde{\Pi}_{L, \geq w}\right) \subseteq \Pi_{M, \geq w_{2}} \\
p_{M, \tau, w_{2}}^{-1}\left(J(\tau)_{\geq w_{1}}^{w_{2}}\right)=J\left(\iota_{L}^{M}\right)\left(J(\tilde{\Pi})_{\geq w}\right) \\
p_{L, \varrho, w_{1}}^{M} \circ p_{M, \tau, w_{2}} \circ J\left(\iota_{L}^{M}\right)=p_{L, \varrho, w} \text { on } J(\tilde{\Pi})_{L, \geq w} .
\end{gathered}
$$

Proof. Let $Q$ be the standard parabolic with Levi $L$. The relation (2.3) follows from the fact that $Q W_{\geq w} U_{0} \subseteq P W_{\geq w_{2}} U_{0}$. The inclusion $\supseteq$ of (2.4) follows from the relation

$$
\left\{m \in M: m w_{2} U_{0} \cap Q W_{\geq w} U_{0} \neq \emptyset\right\}=(M \cap Q) W_{\geq w_{1}}^{M}\left(U_{0} \cap M\right) .
$$

To prove (2.5) we first observe that $w_{1}^{-1} U_{\mathfrak{P}} w_{1}=\left(w_{1}^{-1} U_{\mathfrak{P}} w_{1} \cap M\right) U$ and $U_{\mathfrak{P}}=\left(U_{\mathfrak{P}} \cap M\right) U$, and hence by (2.2) (applied to $w_{2}$ ) we have

$$
w_{2} U_{\mathfrak{P}} w_{2}^{-1} \cap w_{1}^{-1} U_{\mathfrak{P}} w_{1}=\left(w_{2} U_{\mathfrak{P}} w_{2}^{-1} \cap U\right)\left(U_{\mathfrak{P}} \cap w_{1}^{-1} U_{\mathfrak{P}} w_{1} \cap M\right)
$$

and

$$
w_{2} U_{\mathfrak{P}} w_{2}^{-1} \cap U_{\mathfrak{P}}=\left(w_{2} U_{\mathfrak{P}} w_{2}^{-1} \cap U\right)\left(U_{\mathfrak{P}} \cap M\right) .
$$

Let $\varphi \in \tilde{\Pi}_{\geq w}$ and $\varphi^{\prime}=\iota_{L}^{M} \varphi$. Then

$$
\begin{aligned}
\tilde{p}_{L, w}(\varphi) & =\int_{\left(U_{\mathfrak{P}} \cap w^{-1} U_{\mathfrak{P}} w\right) \backslash U_{\mathfrak{P}}} j_{\varrho}(\varphi(w u)) d u \\
& =\int_{\left(U_{\mathfrak{P}} \cap w_{2}^{-1} U_{\mathfrak{P}} w_{2}\right) \backslash U_{\mathfrak{P}}} \int_{\left(U_{\mathfrak{P}} \cap w^{-1} U_{\mathfrak{P}} w\right) \backslash\left(U_{\mathfrak{P}} \cap w_{2}^{-1} U_{\mathfrak{P}} w_{2}\right)} j_{\varrho}\left(\varphi\left(w_{1} w_{2} u_{1} u_{2}\right)\right) d u_{1} d u_{2} \\
& =\int_{\left(U_{\mathfrak{P}} \cap w_{2}^{-1} U_{\mathfrak{P}} w_{2}\right) \backslash U_{\mathfrak{P}}} \int_{\left(w_{2} U_{\mathfrak{P}} w_{2}^{-1} \cap w_{1}^{-1} U_{\mathfrak{P}} w_{1}\right) \backslash\left(w_{2} U_{\mathfrak{P}} w_{2}^{-1} \cap U_{\mathfrak{P}}\right)} j_{\varrho}\left(\varphi\left(w_{1} u_{1} w_{2} u_{2}\right)\right) d u_{1} d u_{2} .
\end{aligned}
$$


Using (2.7) and (2.8) this equals

$$
\begin{aligned}
\int_{\left(U_{\mathfrak{P}} \cap w_{2}^{-1} U_{\mathfrak{P}} w_{2}\right) \backslash U_{\mathfrak{P}}} & \int_{\left(U_{\mathfrak{P}} \cap M \cap w_{1}^{-1} U_{\mathfrak{P}} w_{1}\right) \backslash\left(U_{\mathfrak{P}} \cap M\right)} j_{\varrho}\left(\varphi\left(w_{1} u_{1} w_{2} u_{2}\right)\right) d u_{1} d u_{2} \\
= & \int_{\left(U_{\mathfrak{P}} \cap w_{2}^{-1} U_{\mathfrak{P}} w_{2}\right) \backslash U_{\mathfrak{P}}} \int_{\left(U_{\mathfrak{P}} \cap M \cap w_{1}^{-1} U_{\mathfrak{P}} w_{1}\right) \backslash\left(U_{\mathfrak{P}} \cap M\right)} j_{\varrho}\left(\varphi^{\prime}\left(w_{2} u_{2}\right)\left(w_{1} u_{1}\right)\right) d u_{1} d u_{2} .
\end{aligned}
$$

Note that by $(2.6)$ for any $u_{2} \in U_{0}$ we have $\varphi^{\prime}\left(w_{2} u_{2}\right) \in \tau_{\geq w_{1}}$. Hence we get

$$
\begin{aligned}
\int_{\left(U_{\mathfrak{P}} \cap w_{2}^{-1} U_{\mathfrak{P}} w_{2}\right) \backslash U_{\mathfrak{P}}} \tilde{p}_{L, w_{1}}^{M}\left(\varphi^{\prime}\left(w_{2} u\right)\right) d u=\int_{\left(U_{\mathfrak{P}} \cap w_{2}^{-1} U_{\mathfrak{P}} w_{2}\right) \backslash U_{\mathfrak{P}}} p_{L, w_{1}}^{M}\left(j_{\tau}\left(\varphi^{\prime}\left(w_{2} u\right)\right)\right) d u \\
=p_{L, w_{1}}^{M} \circ \tilde{p}_{M, w_{2}}\left(\varphi^{\prime}\right) .
\end{aligned}
$$

We conclude (2.5).

Finally, we prove (2.4). We already know the inclusion $\supseteq$. First note that $P \tilde{\Omega}_{M,>w_{2}} U_{0}=$ $Q \tilde{\Omega}_{L}^{M} \tilde{\Omega}_{M,>w_{2}} U_{0}$ and hence

Thus,

$$
\Pi_{M,>w_{2}}=\iota_{L}^{M}\left(\tilde{\Pi}_{L, \tilde{\Omega}_{L}^{M} \tilde{\Omega}_{M,>w_{2}}}\right) .
$$

$$
\operatorname{Ker} p_{M, \tau, w_{2}}=J(\Pi)_{M,>w_{2}}=J\left(\iota_{L}^{M}\right)\left(J(\tilde{\Pi})_{L, \tilde{\Omega}_{L}^{M}\left(\tilde{\Omega}_{M}\right)_{>w_{2}}}\right) \subseteq J\left(\iota_{L}^{M}\right)\left(J(\tilde{\Pi})_{L, \geq w}\right) .
$$

Hence, to prove (2.4) it remains to show that

$$
p_{M, \tau, w_{2}} \circ J\left(\iota_{L}^{M}\right)\left(J(\tilde{\Pi})_{\geq w}\right)=J(\tau)_{\geq w_{1}}^{w_{2}} .
$$

We show this for all $w_{1} \in \tilde{\Omega}_{L}^{M}$ by descending induction on $\ell\left(w_{1}\right)$. (Note that (2.5) holds trivially if $w_{1} \in \tilde{\Omega}_{L}^{M} \backslash \Omega_{L}^{M}$.) For the longest element in $\tilde{\Omega}_{L}^{M}$, the equality (2.9) follows from (2.5). For the induction step, (2.5) gives

$$
p_{M, \tau, w_{2}} \circ J\left(\iota_{L}^{M}\right)\left(J(\tilde{\Pi})_{\geq w}\right)+J(\tau)_{>w_{1}}^{w_{2}}=J(\tau)_{\geq w_{1}}^{w_{2}} .
$$

On the other hand, by induction hypothesis we have

$$
p_{M, \tau, w_{2}} \circ J\left(\iota_{L}^{M}\right)\left(J(\tilde{\Pi})_{>w}\right)=J(\tau)_{>w_{1}}^{w_{2}} .
$$

The relation (2.9) follows.

Corollary 3. Under the same assumptions and notation suppose further that $\beta \in \mathscr{C}^{M}$ is a subrepresentation of $\tau$. Identify $I_{M}(\beta)$ (resp. $J\left(I_{M}(\beta)\right)$ ) with a subrepresentation of $\tilde{\Pi}$ (resp. $J(\tilde{\Pi}))$ via $\left(\iota_{L}^{M}\right)^{-1}$ (resp. $\left.J\left(\iota_{L}^{M}\right)^{-1}\right)$. Let $w=w_{1} w_{2} \in \Omega_{L}$ where $w_{1} \in \Omega_{L}^{M}, w_{2} \in \Omega_{M}$. Then the image of

$$
J\left(I_{M}(\beta)\right) \cap J(\tilde{\Pi})_{L, \geq w} \rightarrow J(\tilde{\Pi})_{L, \geq w} / J(\tilde{\Pi})_{L,>w}
$$

is isomorphic to the $w_{2}$-twist of the image of

$$
J(\beta) \cap J(\tau)_{L, \geq w_{1}} \rightarrow J(\tau)_{L, \geq w_{1}} / J(\tau)_{L,>w_{1}} .
$$

In particular if

$$
J(\beta)+J(\tau)_{L,>w_{1}} \supseteq J(\tau)_{L, \geq w_{1}}
$$


then

$$
J\left(I_{M}(\beta)\right)+J(\tilde{\Pi})_{L,>w_{1} w_{2}} \supseteq J(\tilde{\Pi})_{L, \geq w_{1} w_{2}} .
$$

Indeed, the Corollary follows from the relation

$$
\begin{aligned}
p_{L, w}\left(J\left(\iota_{L}^{M}\right)^{-1}\right. & \left.\left(J\left(I_{M}(\beta)\right)\right) \cap J(\tilde{\Pi})_{L, \geq w}\right) \stackrel{(2.5)}{=} p_{L, w_{1}}^{M} \circ p_{M, w_{2}}\left(J\left(I_{M}(\beta)\right) \cap J\left(\iota_{L}^{M}\right)\left(J(\tilde{\Pi})_{L, \geq w}\right)\right) \\
& \stackrel{(2.4)}{=} p_{L, w_{1}}^{M} \circ p_{M, w_{2}}\left(J\left(I_{M}(\beta)\right) \cap p_{M, w_{2}}^{-1}\left(J(\tau)_{\geq w_{1}}^{w_{2}}\right)\right)=p_{L, w_{1}}^{M}\left(J(\beta)^{w_{2}} \cap J(\tau)_{\geq w_{1}}^{w_{2}}\right) .
\end{aligned}
$$

\section{THE GRAPH $\mathscr{E}(\pi)$}

3.1. Ladder representations. As before, we fix a positive integer $d$ and a cuspidal representation $\sigma$ of $G_{d}$.

3.1.1. By a segment $[a, b]$ we mean a set of the form $\{a, a+1, \ldots, b\}$ for some integers $a \leq b$. For any segment $[a, b]$ we denote by $\Delta([a, b])$ the unique irreducible quotient of $\sigma \nu^{a} \times \cdots \times \sigma \nu^{b}$ : it is an essentially square-integrable representation of $G_{d(b-a+1)}$. The map $[a, b] \mapsto \Delta([a, b])$ is a bijection between the set of segments and the set of essentially square-integrable representations in $\operatorname{Irr}_{\sigma}[$ Zel80, §9.3]. By definition, the length $l(\Delta([a, b]))$ of $\Delta([a, b])$ is $b-a+1$. For convenience, by a slight abuse of notation, we set $\Delta([a, a-1])=1$ (the one-dimensional representation of $G_{0}$ ) for any $a \in \mathbb{Z}$ and $\Delta([a, b])=0$ (in $\mathscr{R}$ ) for $b<a-1$.

Let $\left[a_{1}, b_{1}\right], \ldots,\left[a_{t}, b_{t}\right]$ be segments and let $\Delta_{i}=\Delta\left(\left[a_{i}, b_{i}\right]\right)$ and $m=\sum_{i} l\left(\Delta_{i}\right)$. Assume that for all $i<j, \Delta_{i}$ does not precede $\Delta_{j}$, i.e. we do not have $a_{i}<a_{j} \leq b_{i}+1<b_{j}+1$ for $i<j$. Then the representation

$$
I\left(\Delta_{1}, \ldots, \Delta_{t}\right):=\Delta_{1} \times \cdots \times \Delta_{t}
$$

admits a unique irreducible quotient, the Langlands quotient, which we denote by

$$
\mathrm{L}\left(\Delta_{1}, \ldots, \Delta_{t}\right)
$$

(see for example [Rod82, Théorème 3]). Any element of $\operatorname{Irr}_{\sigma}$ is of this form, for uniquely determined $\Delta_{1}, \ldots, \Delta_{t}$ (up to permutation). More generally, by reordering $\Delta_{1}, \ldots, \Delta_{t}$ if necessary, we define $\mathrm{L}\left(\Delta_{1}, \ldots, \Delta_{t}\right)$ for any $t$-tuple.

3.1.2. Let $\left[a_{1}, b_{1}\right], \ldots,\left[a_{t}, b_{t}\right]$ be segments and let $\Delta_{i}=\Delta\left(\left[a_{i}, b_{i}\right]\right)$. We say that $\Delta_{1}, \ldots, \Delta_{t}$ form a ladder if $a_{1}>\cdots>a_{t}$ and $b_{1}>\cdots>b_{t}$. In this case we term $\mathrm{L}\left(\Delta_{1}, \ldots, \Delta_{t}\right)$ a ladder representation. A particularly important special case is the class of Speh representations for which $a_{i+1}=a_{i}-1$ and $b_{i+1}=b_{i}-1$ for all $i=1, \ldots, t-1$. These representations comprise the building blocks for the unitary dual of $G_{n}([\operatorname{Tad} 86])$.

3.2. Let $\left[a_{1}, b_{1}\right], \ldots,\left[a_{t}, b_{t}\right]$ be segments, $\Delta_{i}=\Delta\left(\left[a_{i}, b_{i}\right]\right)$ and $m=\sum_{i} l\left(\Delta_{i}\right)$. Let $\pi=$ $\mathrm{L}\left(\Delta_{1}, \ldots, \Delta_{t}\right) \in \operatorname{Irr} G_{m d}$. We order the segments so that for all $i<j$ we have $b_{i} \geq b_{j}$ and if $b_{i}=b_{j}$ then $a_{i} \geq a_{j}$. (In the sequel we only consider the case where $b_{1}>\cdots>b_{t}$.) 
(1) Define $\mathscr{I}(\pi)$ to be the directed graph whose vertex set is

$$
\mathfrak{S}\left(\left[a_{1}, b_{1}\right], \ldots,\left[a_{t}, b_{t}\right]\right)=\left\{(j, i): i=1, \ldots, t, j \in\left[a_{i}, b_{i}\right]\right\}
$$

and the edges are given by the horizontal arrows

$$
(j, i) \prec(j-1, i), i=1, \ldots, t, j \in\left[a_{i}+1, b_{i}\right] .
$$

(2) Suppose moreover that $\pi$ is a ladder representation. Denote by $\mathscr{E}(\pi)$ the directed graph obtained from $\mathscr{I}(\pi)$ by inserting the additional edges (diagonal arrows)

$$
(j, i+1) \prec(j+1, i), i=1, \ldots, t-1, j \in\left[a_{i}-1, b_{i+1}\right] .
$$

Example 1. Suppose that $\Delta_{1}=\Delta([2,6]), \Delta_{2}=\Delta([0,5]), \Delta_{3}=\Delta([-3,0])$. Then the graph $\mathscr{I}\left(\mathrm{L}\left(\Delta_{1}, \Delta_{2}, \Delta_{3}\right)\right)$ is

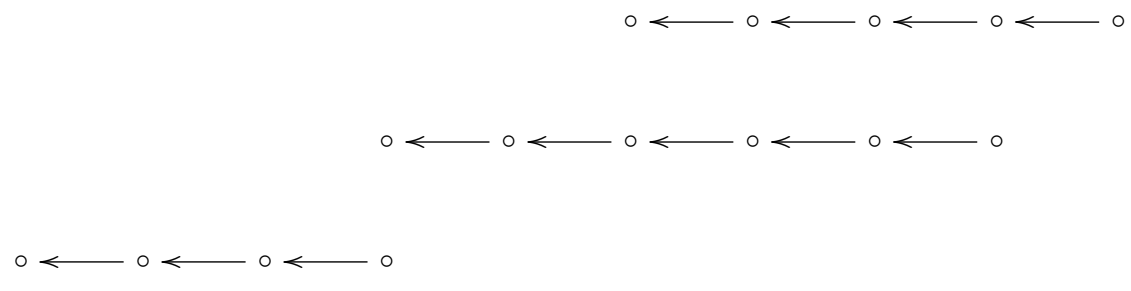

and the graph $\mathscr{E}\left(\mathrm{L}\left(\Delta_{1}, \Delta_{2}, \Delta_{3}\right)\right)$ is

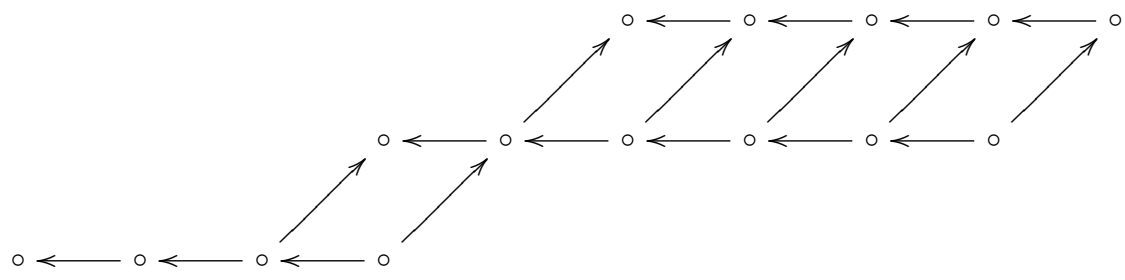

The graph $\mathscr{E}(\pi)$ will play a role in the next section where we will relate it to $\mathbf{J}(\pi)$. It is motivated by the combinatorial description of the Zelevinsky involution $\pi^{\dagger}$ [Zel80, §9] à la Mœglin-Waldspurger [MW86] which takes a particularly simple form in the case of ladder representations. Indeed, in terms of the graph $\mathscr{I}(\pi)$, the segments pertaining to $\pi^{\dagger}$ are formed by intersecting with the diagonals $i+j=$ const. In other words, we can think of the diagonal arrows in $\mathscr{E}(\pi)$ as the horizontal arrows of the segments of $\pi^{\dagger}$. In particular, it is easy to see that the class of ladder representations (as well as the Speh representations) is closed under the Zelevinsky involution. 
Example 2. Suppose again that $\Delta_{1}=\Delta([2,6]), \Delta_{2}=\Delta([0,5]), \Delta_{3}=\Delta([-3,0])$. We write the segments

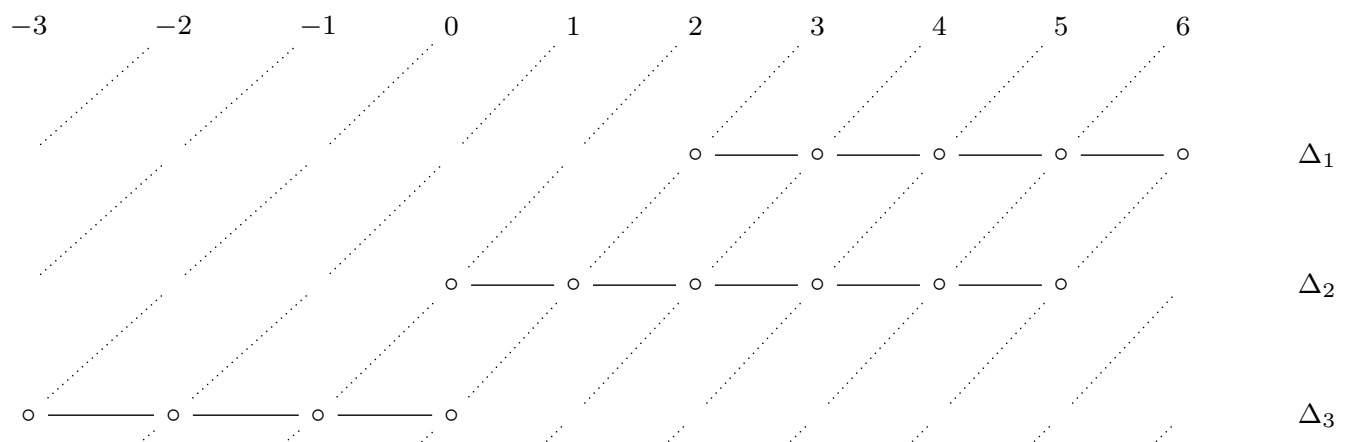

and, by intersecting with diagonals, we get

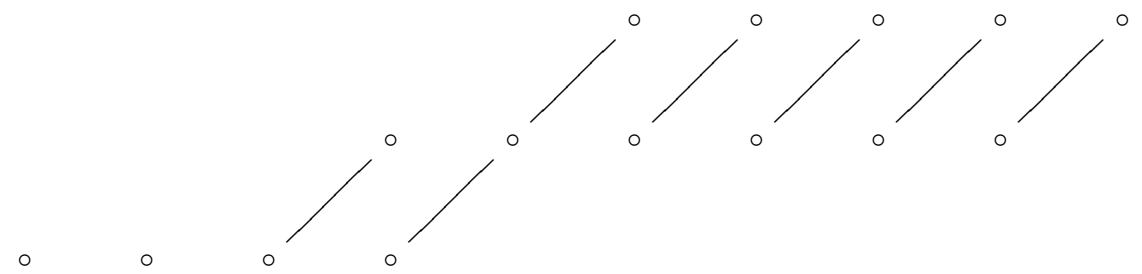

that is, the Zelevinsky dual multisegment

$$
([5,6],[4,5],[3,4],[2,3],[0,2],[-1,0],[-2,-2],[-3,-3]) .
$$

3.3. Let $\mathscr{G}=(\mathscr{V}, \prec)$ be a finite directed graph with $|\mathscr{V}|=m$. A labelling of $\mathscr{V}$ (or of $\mathscr{G}$ ) is a bijection $\phi: \mathscr{V} \rightarrow\{1, \ldots, m\}$. We denote by $\mathcal{N}(\mathscr{G})$ the set of all labellings of $\mathscr{G}$. Thus, $|\mathcal{N}(\mathscr{G})|=m$ !. We say that a labelling $\phi$ is increasing if $\phi\left(v_{1}\right)<\phi\left(v_{2}\right)$ whenever $v_{1} \prec v_{2}$. Let $\mathcal{M}(\mathscr{G})$ be the set of increasing labellings of $\mathscr{G}$.

When $\mathscr{V} \subseteq \mathbb{R}^{2}$, for any $\phi \in \mathcal{N}(\mathscr{G})$ we write $\lambda_{\phi}=p_{1} \circ \phi^{-1}:\{1, \ldots, m\} \rightarrow \mathbb{R}$ where $p_{1}: \mathbb{R}^{2} \rightarrow \mathbb{R}$ is the projection in the first coordinate. We call $\lambda_{\phi}$ the weight of $\phi$.

Example 3. Let $\left[a_{1}, b_{1}\right], \ldots,\left[a_{t}, b_{t}\right]$ be segments, $\Delta_{i}=\Delta\left(\left[a_{i}, b_{i}\right]\right), M$ the Levi subgroup of type $m_{1} d, \ldots, m_{t} d$ where $m_{i}=l\left(\Delta_{i}\right)$ and $\pi=\mathrm{L}\left(\Delta_{1}, \ldots, \Delta_{t}\right) \in \operatorname{Irr} G_{m d}$ where $m=\sum m_{i}$. Assume that $a_{1}>\cdots>a_{t}$. We define the "standard labelling" $\phi_{\text {std }}=\phi_{\text {std }, \pi} \in \mathcal{M}(\mathscr{I}(\pi))$ to be the one given by

$$
\begin{aligned}
\phi_{\text {std }}\left(b_{1}, 1\right)=1<\cdots<\phi_{\text {std }}\left(a_{1}, 1\right)<\phi_{\text {std }}\left(b_{2}, 2\right)< & \cdots<\phi_{\text {std }}\left(a_{2}, 2\right)< \\
& \cdots<\phi_{\text {std }}\left(b_{t}, t\right)<\cdots<\phi_{\text {std }}\left(a_{t}, t\right)=m .
\end{aligned}
$$


For instance, in the previous examples $\phi_{\text {std }}$ is the labelling

$$
\begin{aligned}
& { }_{0}^{5} \longleftarrow{ }_{0}^{4} \longleftarrow{ }_{0}^{3} \longleftarrow{ }_{0}^{2} \longleftarrow{ }_{0}^{1} \\
& \underset{0}{11} \longleftarrow{ }_{0}^{10} \longleftarrow{ }_{0}^{9} \longleftarrow{ }_{0}^{8} \longleftarrow{ }_{0}^{7} \longleftarrow{ }_{0}^{6} \\
& \underset{0}{15} \longleftarrow \underset{0}{14} \longleftarrow{ }_{0}^{13} \longleftarrow{ }_{0}^{12}
\end{aligned}
$$

By $§ 2.6$, there is a bijection

$$
\begin{aligned}
\psi_{\pi}: \Omega_{M} & \longrightarrow \mathcal{M}(\mathscr{I}(\pi)) \\
w & \mapsto w^{-1} \circ \phi_{\text {std }}
\end{aligned}
$$

In particular, $|\mathcal{M}(\mathscr{I}(\pi))|=\left|\Omega_{M}\right|=\left(\begin{array}{ccc}m \\ m_{1} & m_{2} & \ldots \\ m_{t}\end{array}\right)$.

Remark 2. Combinatorially, $\psi_{\pi}^{-1}\left(\phi_{1}\right) \leq \psi_{\pi}^{-1}\left(\phi_{2}\right)$ in the Bruhat order if and only for any $k=1, \ldots, t-1$ if we write $\left\{\phi_{1}(j, i): j \in\left[a_{i}, b_{i}\right], i \leq k\right\}=\left\{x_{1}, \ldots, x_{l}\right\}$ with $x_{1}<\cdots<x_{l}$ and $\left\{\phi_{2}(j, i): j \in\left[a_{i}, b_{i}\right], i \leq k\right\}=\left\{y_{1}, \ldots, y_{l}\right\}$ with $y_{1}<\cdots<y_{l}$ then $x_{i} \leq y_{i}$ for all $i=1, \ldots, l$. (Cf. [BB05, Theorem 2.1.5].)

Remark 3. In the ladder case, with the terminology of [Sta99], $\mathcal{M}(\mathscr{E}(\pi))$ is in bijection with the standard Young Tableaux of the skew Young diagram $\left(a_{1}+1, \ldots, a_{t}+t\right) /\left(b_{1}+\right.$ $\left.1, \ldots, b_{t}+t\right)$. In particular, as a consequence of the Jacobi-Trudi identity we have

$$
|\mathcal{M}(\mathscr{E}(\pi))|=m ! \operatorname{det}\left(\frac{1}{\left(b_{i}-a_{j}+1\right) !}\right)_{i, j=1, \ldots, t}
$$

where we set $k !=\infty$ for $k<0$ (cf. [Sta99, Corollary 7.16.3]).

3.4. Suppose $\pi$ is a ladder representation. We evidently have $\mathcal{M}(\mathscr{E}(\pi)) \subseteq \mathcal{M}(\mathscr{I}(\pi))$. Define $\Omega_{\pi} \subseteq \Omega_{M}$ to be the inverse image of $\mathcal{M}(\mathscr{E}(\pi))$ under $\psi_{\pi}$.

Next, we will characterize the increasing labellings in $\mathcal{M}(\mathscr{E}(\pi))$ in terms of their weight.

Definition 1. We say that $\phi \in \mathcal{M}(\mathscr{I}(\pi))$ is a G-T pattern if $\lambda=\lambda_{\phi}$ satisfies the following two conditions:

(1) Whenever $\lambda(x)=\lambda(y)$ with $x<y$, there exists $x<z<y$ such that $\lambda(z)=\lambda(x)-1$

(2) for any $i=2, \ldots, t$ the smallest $x$ such that $\lambda(x) \in\left\{b_{i}, b_{i}+1\right\}$ satisfies $\lambda(x)=b_{i}$.

Of course this definition is closely related to Gelfand-Tsetlin patterns (e.g., [Bum04, p. 359]). For $t=2$ we can rephrase the condition by saying that there does not exist $l \in\left[a_{1}, b_{2}+1\right]$ such that $\lambda(i)=\lambda(j)+1=l$ implies $i<j$.

Lemma 4. Let $\phi \in \mathcal{M}(\mathscr{I}(\pi))$. Then $\phi \in \mathcal{M}(\mathscr{E}(\pi))$ if and only if $\phi$ is a $G$-T pattern.

Proof. 
Suppose first that $\phi \in \mathcal{M}(\mathscr{E}(\pi))$. Fix $c \in\left[a_{t}, b_{1}\right]$ and $c-1 \quad c$ let $i_{1}$ (resp. $i_{2}$ ) be the minimal (resp. maximal) index $i$ such that $c \in\left[a_{i}, b_{i}\right]$. If $i_{1}<i_{2}$, then $\phi\left(c, i_{2}\right)<\phi(c-$ $\left.1, i_{2}\right)<\phi\left(c, i_{2}-1\right)<\phi\left(c-1, i_{2}-1\right)<\cdots<\phi\left(c, i_{1}\right)$. This shows the first condition. The second condition follows by noting that $x=\phi\left(b_{i}, i\right)$ since the minimal $y$ such that $\chi(y)=b_{i}+1$ is $\phi\left(b_{i}+1, i-1\right)$. Thus, $\phi$ is a G-T pattern. Conversely, suppose that $\phi \in \mathcal{M}(\mathscr{I}(\pi)) \backslash \mathcal{M}(\mathscr{E}(\pi))$ and let $\left(j_{0}, i_{0}\right)$ be the maximal pair $(j, i), i=1, \ldots, t-1$, $j \in\left[a_{i}-1, b_{i+1}\right]$ with respect to the lexicographic order (from left to right) such that $\phi(j+1, i)<\phi(j, i+1)$. There are two possibilities: either $j_{0}<b_{i_{0}+1}$ or $j_{0}=b_{i_{0}+1}$.

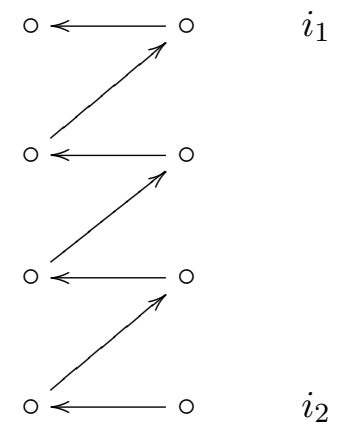

In the first case let $x=\phi\left(j_{0}+1, i_{0}+1\right)$, and $y=\phi\left(j_{0}+1, i_{0}\right)$ and observe that by the maximality of $j_{0}$ we have

$$
y>\phi\left(j_{0}+2, i_{0}\right)>x .
$$

Suppose that $i$ is such that $j_{0} \in\left[a_{i}, b_{i}\right]$. If $i>i_{0}+1$ then by maximality of $\left(j_{0}, i_{0}\right)$ we have

$$
\phi\left(j_{0}, i\right)<\phi\left(j_{0}+1, i-1\right) \leq \phi\left(j_{0}+i-i_{0}-1, i_{0}+1\right) \leq \phi\left(j_{0}+1, i_{0}+1\right)=x .
$$

Similarly, if $i \leq i_{0}$ then

$$
y=\phi\left(j_{0}+1, i_{0}\right) \leq \phi\left(j_{0}+i_{0}+1-i, i\right)<\phi\left(j_{0}, i\right) .
$$

Also $y<\phi\left(j_{0}, i_{0}+1\right)$ by the defining property of $\left(j_{0}, i_{0}\right)$. Thus the first condition of G-T pattern is violated.

On the other hand, in the case where $j_{0}=b_{i_{0}+1}$ we have $\phi\left(j_{0}, i_{0}+1\right)>\phi\left(j_{0}+1, i_{0}\right)$ and $\phi\left(j_{0}, i\right)>\phi\left(j_{0}+1, i\right)$ for all $i \leq i_{0}$ such that $j_{0} \in\left[a_{i}, b_{i}\right]$. Therefore the second condition of $\mathrm{G}-\mathrm{T}$ pattern is violated for $i_{0}+1$.

3.5. For any function $\lambda:\{1, \ldots, m\} \rightarrow \mathbb{R}$ let

$$
\sigma[\lambda]=\sigma \nu^{\lambda(1)} \otimes \cdots \otimes \sigma \nu^{\lambda(m)} \in \operatorname{Irr} M_{\mathfrak{P}}
$$

For any finite directed graph $\mathscr{G}=(\mathscr{V}, \prec)$ with $m$ vertices contained in $\mathbb{R}^{2}$ and any subset $A \subseteq \mathcal{N}(\mathscr{G})$ let

$$
\mathbf{X}_{A}=\sum_{\phi \in A} \sigma\left[\lambda_{\phi}\right] \in \mathscr{R}\left(M_{\mathfrak{P}}\right)
$$

where we recall that $\lambda_{\phi}$ is the weight of $\phi$. For example, by the geometric Lemma of Bernstein-Zelevinsky [Zel80, §1.2], with the notations of the previous paragraph, we have

$$
\mathbf{J}\left(\Delta_{1} \times \cdots \times \Delta_{t}\right)=\mathbf{X}_{\mathcal{M}(\mathscr{I}(\pi))} .
$$


3.6. Let $\pi$ be a ladder representation. Since

$$
\mathcal{M}(\mathscr{E}(\pi)) \subseteq \mathcal{M}(\mathscr{I}(\pi))
$$

we get

$$
\mathbf{X}_{\mathcal{M}(\mathscr{E}(\pi))} \leq \mathbf{X}_{\mathcal{M}(\mathscr{I}(\pi))}
$$

The following is an immediate consequence of Lemma 4 and the definition of $\mathbf{X}_{\mathcal{M}(\mathscr{G}) \text {. }}$

Corollary 5. $\mathbf{X}_{\mathcal{M}(\mathscr{E}(\pi))}$ and $\mathbf{X}_{\mathcal{M}(\mathscr{I}(\pi))}-\mathbf{X}_{\mathcal{M}(\mathscr{E}(\pi))}$ are disjoint.

\section{The MAin TheOREM}

Throughout this section we fix segments $\left[a_{1}, b_{1}\right], \ldots,\left[a_{t}, b_{t}\right]$ and set $\Delta_{i}=\Delta\left(\left[a_{i}, b_{i}\right]\right)$, $i=1, \ldots, t$. We assume that $\Delta_{1}, \ldots, \Delta_{t}$ form a ladder and let $\delta=\Delta_{1} \otimes \cdots \otimes \Delta_{t}$ and $\pi=\mathrm{L}\left(\Delta_{1}, \ldots, \Delta_{t}\right)$. Let $M$ be the Levi subgroup of type $d\left(b_{1}-a_{1}+1\right), \ldots, d\left(b_{t}-a_{t}+1\right)$ so that $\delta$ is a representation of $M$. Denote by $\mathscr{L}$ the (unique) maximal submodule of $\Delta_{1} \times \cdots \times \Delta_{t}=I_{M}(\delta)$ so that $\pi=I_{M}(\delta) / \mathscr{L}$. We write $\mathscr{J}=J\left(I_{M}(\delta)\right)$, so that $[\mathscr{J}]=$ $\mathbf{J}\left(I_{M}(\delta)\right)$ is equal by $(3.2)$ to $\mathbf{X}_{\mathcal{M}(\mathscr{I}(\pi))}$. (Recall that $J$ denotes Jacquet functor with respect to the parabolic of type $(d, \ldots, d)$.)

4.1. For any subset $A \subseteq \mathcal{M}(\mathscr{I}(\pi))$ (cf. $\S 3.3)$ let $\mathscr{J}^{A}$ denote the maximal quotient of $\mathscr{J}$ such that all its composition factors are equivalent to $\sigma\left[\lambda_{\phi}\right]$ for some $\phi \in A$. Let $\mathscr{J}_{A}$ be the submodule so that $\mathscr{J}^{A}=\mathscr{J} / \mathscr{J}_{A}$. Since $\mathscr{J}$ is cuspidal and $[\mathscr{J}]=\mathbf{X}_{\mathcal{M}(\mathscr{I}(\pi))}$, we have the following properties.

Lemma 6. (1) $\mathscr{J}^{A}$ is the quotient of $\mathscr{J}$ characterized by $\left[\mathscr{J}^{A}\right]=\mathbf{X}_{A}$.

(2) $\mathscr{J}_{A}$ is the maximal submodule of $\mathscr{J}$ such that none of its composition factors is equivalent to $\sigma\left[\lambda_{\phi}\right]$ for any $\phi \in A$.

(3) $\mathscr{J}_{A}$ is the submodule of $\mathscr{J}$ characterized by $\left[\mathscr{J}_{A}\right]=\mathbf{X}_{\mathcal{M}(\mathscr{I}(\pi)) \backslash A}\left(=\mathbf{X}_{\mathcal{M}(\mathscr{I}(\pi))}-\mathbf{X}_{A}\right)$.

(4) $\mathscr{J}^{A}$ and $\mathscr{J}_{A}$ are disjoint.

(5) We have a direct sum decomposition $\mathscr{J}=\mathscr{J}_{A}+\mathscr{J}_{\mathcal{M}(\mathscr{I}(\pi)) \backslash A}$. Hence $\mathscr{J}^{A} \simeq$ $\mathscr{J}_{\mathcal{M}(\mathscr{I}(\pi)) \backslash A}$

(6) More generally for any submodule $M$ of $\mathscr{J}$ we have $M=\left(M \cap \mathscr{J}_{A}\right)+(M \cap$

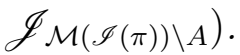

(7) For any submodules $M_{1}, M_{2}$ of $\mathscr{J}$ we have

$$
\left(M_{1}+M_{2}\right) \cap \mathscr{J}_{A}=\left(M_{1} \cap \mathscr{J}_{A}\right)+\left(M_{2} \cap \mathscr{J}_{A}\right)
$$

(8) Suppose that $M_{1} \subseteq M_{2} \subseteq \mathscr{J}$ and $M_{2} / M_{1} \simeq \sigma[\lambda]$ where $\lambda \notin A$. Then $M_{1}+\mathscr{J}_{A} \supseteq$ $M_{2}$. 
4.2. For any $w \in S_{t}$ let $\mathcal{I}_{w}=\Delta\left(\left[a_{w(1)}, b_{1}\right]\right) \times \cdots \times \Delta\left(\left[a_{w(t)}, b_{t}\right]\right)$. (Note that this is 0 unless $a_{w(i)} \leq b_{i}+1$ for all $i$.) In particular, let $\mathscr{K}_{k}=\mathcal{I}_{s_{k}}$ where $s_{k}$ is the transposition $(k, k+1)$. We can identify $\mathscr{K}_{k}$ with a submodule of $\Delta_{1} \times \cdots \times \Delta_{t}$. Let

$$
\mathscr{K}:=\sum_{k=1}^{t-1} \mathscr{K}_{k} \subseteq \Delta_{1} \times \cdots \times \Delta_{t} .
$$

Note that if $w_{1} \leq w_{2}$ in the Bruhat order then $\left[\mathcal{I}_{w_{2}}\right] \leq\left[\mathcal{I}_{w_{1}}\right]$. In particular, for any $w \neq \mathrm{Id}$ there exists $k$ such that $\left[\mathcal{I}_{w}\right] \leq\left[\mathscr{K}_{k}\right]$ and hence

$$
\left[\mathcal{I}_{w}\right] \leq[\mathscr{K}]
$$

Theorem 7. Assume that $\Delta_{1}, \ldots, \Delta_{t}$ is a ladder and let $\pi=\mathrm{L}\left(\Delta_{1}, \ldots, \Delta_{t}\right)$. Then

(1) $J(\mathscr{K})=\mathscr{J}_{\mathcal{M}(\mathscr{E}(\pi))}$.

(2) $J(\pi)=\mathscr{J}^{\mathcal{M}(\mathscr{E}(\pi))}$. Equivalently (by Lemma 6 and Corollary 5),

$$
\mathbf{J}(\pi)=\mathbf{X}_{\mathcal{M}(\mathscr{E}(\pi))}=\sum_{\phi \in \mathcal{M}(\mathscr{E}(\pi))} \sigma \nu^{\lambda_{\phi}(1)} \otimes \cdots \otimes \sigma \nu^{\lambda_{\phi}(m)} .
$$

(3) $\mathscr{K}=\mathscr{L}$.

In particular, $J(\pi)$ and $J(\mathscr{K})$ are disjoint.

Before proving the theorem we note that any two parts of it imply the third.

4.3. The maximal case. Consider first the case $t=2$. Recall that in this case

$$
\Delta_{1} \times \Delta_{2}=\pi+\mathscr{K}
$$

in $\mathscr{R}$ and $\mathscr{K}=\mathscr{L}=\Delta_{1}^{\prime} \times \Delta_{2}^{\prime}$ where $\Delta_{1}^{\prime}=\Delta\left(\left[a_{2}, b_{1}\right]\right)$ and $\Delta_{2}^{\prime}=\Delta\left(\left[a_{1}, b_{2}\right]\right)$. If $a_{1}>b_{2}+1$ then $\pi=I_{M}(\delta), \mathscr{K}=\mathscr{L}=0$ and Theorem 7 is trivial in that case. Suppose from now on that $a_{1} \leq b_{2}+1$ and let $\mathscr{G}_{1}=\mathscr{I}(\mathscr{L})$ and $\mathscr{G}=\mathscr{I}(\pi)$.

We will introduce a weight preserving injective map $\iota_{1}: \mathcal{M}\left(\mathscr{G}_{1}\right) \rightarrow \mathcal{M}(\mathscr{G})$ whose image is the complement of $\mathcal{M}(\mathscr{E}(\pi))$.

To define it, let $\phi \in \mathcal{M}\left(\mathscr{G}_{1}\right)$. For convenience we set $\phi\left(a_{1}-1,2\right)=\infty$. Let $j_{0}$ be the largest $j \in\left[a_{1}, b_{2}+1\right]$ such that $\phi(j, 1)<\phi(j-1,2)$. Clearly, the map

$$
\kappa_{j_{0}}(j, i)= \begin{cases}(j, 3-i) & \text { if } j<j_{0} \\ (j, i) & \text { otherwise }\end{cases}
$$

defines a bijection between the vertices of $\mathscr{G}$ and $\mathscr{G}_{1}$. 

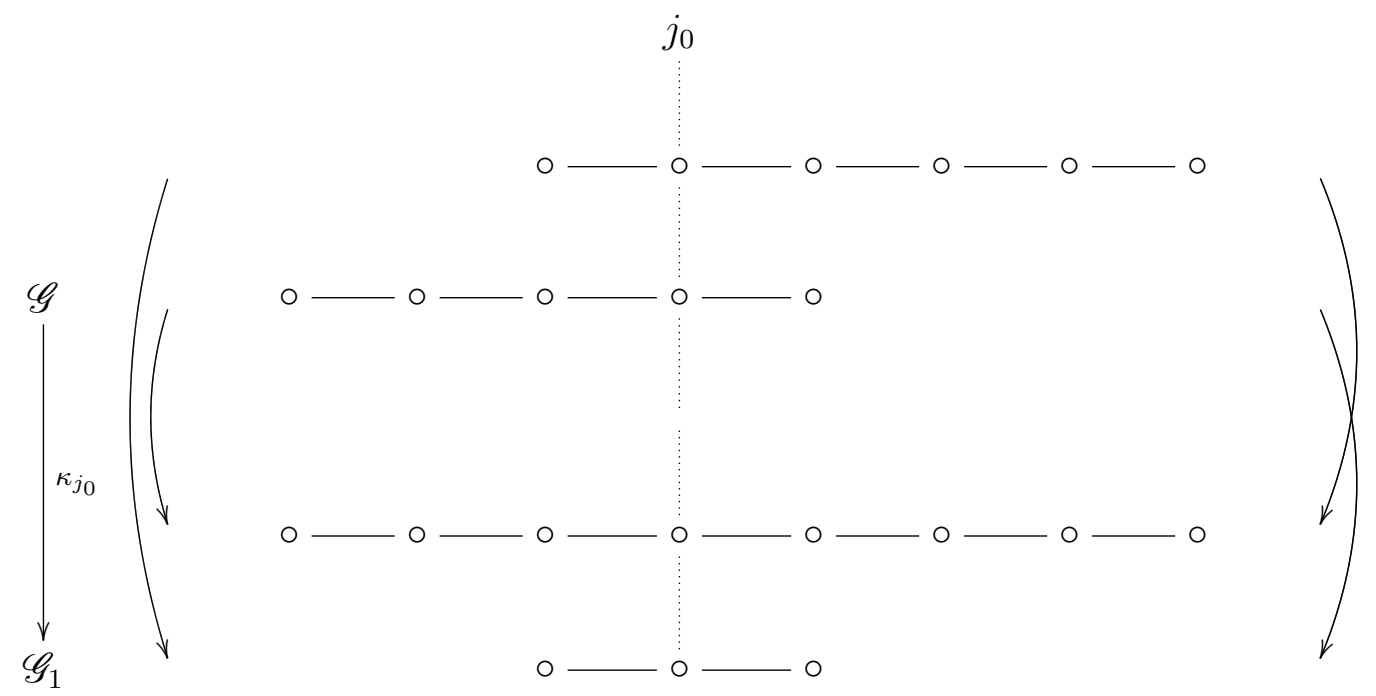

We define $\phi^{\prime}=\iota_{1}(\phi): \mathscr{G} \rightarrow\{1, \ldots, m\}$ by $\phi^{\prime}=\phi \circ \kappa_{j_{0}}$. Evidently $\lambda_{\phi^{\prime}}=\lambda_{\phi}$. To check that $\phi^{\prime} \in \mathcal{M}(\mathscr{G})$ we observe that by assumption on $j_{0}$

$$
\phi^{\prime}\left(j_{0}, 1\right)=\phi\left(j_{0}, 1\right)<\phi\left(j_{0}-1,2\right)=\phi^{\prime}\left(j_{0}-1,1\right)
$$

and (if $j_{0} \leq b_{2}$ )

$$
\phi^{\prime}\left(j_{0}, 2\right)=\phi\left(j_{0}, 2\right)<\phi\left(j_{0}+1,1\right)<\phi\left(j_{0}, 1\right)<\phi\left(j_{0}-1,1\right)=\phi^{\prime}\left(j_{0}-1,2\right)
$$

where the first inequality follows from the maximality of $j_{0}$. The other inequalities

$$
\phi^{\prime}(j, i)<\phi^{\prime}(j-1, i) \quad j_{0} \neq j \in\left[a_{i}+1, b_{i}\right]
$$

follow directly from the corresponding inequalities for $\phi$.

Finally, the inverse map $\mathcal{M}(\mathscr{G}) \backslash \mathcal{M}(\mathscr{E}(\pi)) \rightarrow \mathcal{M}\left(\mathscr{G}_{1}\right)$ is defined in exactly the same way. (The index $j_{0}$ is well-defined for $\phi \in \mathcal{M}(\mathscr{G}) \backslash \mathcal{M}(\mathscr{E}(\pi))$.) We infer that

$$
\mathbf{J}(\mathscr{K})=\mathbf{X}_{\mathcal{M}\left(\mathscr{G}_{1}\right)}=\mathbf{X}_{\mathcal{M}(\mathscr{G}) \backslash \mathcal{M}(\mathscr{E}(\pi))}=\mathbf{X}_{\mathcal{M}(\mathscr{G})}-\mathbf{X}_{\mathcal{M}(\mathscr{E}(\pi))}
$$

and

$$
\mathbf{J}(\pi)=\mathbf{X}_{\mathcal{M}(\mathscr{E}(\pi))}
$$

As was pointed out before, the first part of Theorem 7 in the case $t=2$ now follows from the disjointness of $\mathbf{X}_{\mathcal{M}(\mathscr{E}(\pi))}$ and $\mathbf{X}_{\mathcal{M}(\mathscr{G})}-\mathbf{X}_{\mathcal{M}(\mathscr{E}(\pi))}$.

For future record we say that $\phi^{\prime}$ as above is obtained from $\phi$ by a flip-flop along the two rows. Of course, for this procedure we do not need to assume necessarily that $\phi$ takes values in $\{1, \ldots, m\}$.

Recall the bijection $\psi_{\pi}: \Omega_{M} \longrightarrow \mathcal{M}(\mathscr{G})$ defined in (3.1). We further note that for any $w \in \Omega_{M}$ such that $\psi_{\pi}(w) \in \iota_{1}\left(\mathcal{M}\left(\mathscr{G}_{1}\right)\right)$ we have

$$
\mathscr{J}_{>w}+\mathscr{K} \supseteq \mathscr{J}_{\geq w}
$$

This follows from Lemma 6 because $\mathscr{J}_{\geq w} / \mathscr{J}_{>w} \simeq \sigma\left[\lambda_{\psi_{\pi}(w)}\right], \psi_{\pi}(w) \notin \mathcal{M}(\mathscr{E}(\pi))$ and $\mathscr{K}=\mathscr{J}_{\mathcal{M}(\mathscr{E}(\pi))}$ by what we just proved. 
4.4. The general case. Let $P_{k}, k=1, \ldots, t-1$ be the parabolic subgroup of type $\left(n_{1}, \ldots, n_{k-1}, n_{k}+n_{k+1}, n_{k+2}, \ldots, n_{t}\right)$. Recall that, by definition, $\mathscr{K}_{k}$ is isomorphic to $I_{M_{k}}\left(\varrho_{k}\right)$ where

$$
\varrho_{k}=\Delta_{1} \otimes \cdots \otimes \Delta_{k-1} \otimes \Delta\left(\left[a_{k+1}, b_{k}\right]\right) \times \Delta\left(\left[a_{k}, b_{k+1}\right]\right) \otimes \Delta_{k+2} \otimes \cdots \otimes \Delta_{t} .
$$

Let

$$
\mathscr{G}_{k}=\mathscr{I}\left(\mathrm{L}\left(\Delta_{1}, \ldots, \Delta_{k-1}, \Delta\left(\left[a_{k+1}, b_{k}\right]\right), \Delta\left(\left[a_{k}, b_{k+1}\right]\right), \Delta_{k+2}, \ldots, \Delta_{t}\right)\right) .
$$

(If $a_{k}>b_{k+1}+1$ then we set $\mathscr{G}_{k}=\emptyset$.) Using the map $\iota_{1}$ defined above in the case $t=2$ we define an injective map $\iota_{k}: \mathcal{M}\left(\mathscr{G}_{k}\right) \rightarrow \mathcal{M}(\mathscr{G})$ by flip-flop along rows $k, k+1$. More precisely we define $\iota_{k}(\phi)=\phi \circ \kappa_{k, j_{0}}$ for $\phi \in \mathcal{M}\left(\mathscr{G}_{k}\right)$ where $j_{0}$ is the largest $j \in\left[a_{k}, b_{k+1}+1\right]$ such that $\phi(j, k)<\phi(j-1, k+1)$ (with $\left.\phi\left(a_{k}-1, k+1\right)=\infty\right)$ and $\kappa_{k, j_{0}}: \mathscr{G} \rightarrow \mathscr{G}_{k}$ is the bijection

$$
\kappa_{k, j_{0}}(j, i)= \begin{cases}\left(j, s_{k}(i)\right) & \text { if } j<j_{0}, \\ (j, i) & \text { otherwise. }\end{cases}
$$

The map $\iota_{k}$ is weight-preserving and therefore

$$
\mathbf{J}\left(\mathscr{K}_{k}\right)=\mathbf{J}\left(I_{M_{k}}\left(\varrho_{k}\right)\right)=\mathbf{X}_{\mathcal{M}\left(\mathscr{G}_{k}\right)}=\mathbf{X}_{\mathcal{M}_{k}(\mathscr{G})}
$$

where $\mathcal{M}_{k}(\mathscr{G})$ is the image of $\iota_{k}$.

We can characterize $\mathcal{M}_{k}(\mathscr{G})$ as the set of elements $\phi$ in $\mathcal{M}(\mathscr{G})$ for which $\phi(j, k)<$ $\phi(j-1, k+1)$ for some $j \in\left[a_{k}, b_{k+1}+1\right]$. It follows from the definition of $\mathcal{M}(\mathscr{E}(\pi))$ that $\cup_{k=1}^{t-1} \mathcal{M}_{k}(\mathscr{G})$ is the complement of $\mathcal{M}(\mathscr{E}(\pi))$ in $\mathcal{M}(\mathscr{G})$.

We conclude from Lemma 4 that $J\left(\mathscr{K}_{k}\right) \subseteq \mathscr{J}_{\mathcal{M}(\mathscr{E}(\pi))}$ for all $k$, and hence $J(\mathscr{K})=$ $\sum_{k=1}^{t} J\left(\mathscr{K}_{k}\right) \subseteq \mathscr{J}_{\mathcal{M}(\mathscr{E}(\pi))}$

To conclude the first part of Theorem 7 it remains to show the following

Lemma 8. $J(\mathscr{K}) \supseteq \mathscr{J}_{\mathcal{M}(\mathscr{E}(\pi))}$.

Proof. First note that the condition $\psi_{\pi}(w) \in \mathcal{M}_{k}(\mathscr{G})$ depends only on the right $\Omega_{M_{k}}$-coset of $w$. Hence

$$
\psi_{\pi}^{-1}\left(\mathcal{M}_{k}(\mathscr{G})\right)=\omega_{k} \Omega_{M_{k}}
$$

where $\omega_{k}=\Omega_{M}^{M_{k}} \cap \psi_{\pi}^{-1}\left(\mathcal{M}_{k}(\mathscr{G})\right.$ ). Therefore, $\Omega_{\pi}$ (see $\S 3.4$ ) is the complement of $\cup_{k=1}^{t-1} \omega_{k} \Omega_{M_{k}}$ in $\Omega_{M}$.

We will show by descending induction on $\ell(w)$ that $\mathscr{J}_{\mathcal{M}(\mathscr{E}(\pi))} \cap \mathscr{J}_{\geq w} \subseteq J(\mathscr{K})$ for all $w \in \Omega_{M}$. For the induction step (as well as for the base of the induction) we may assume by (4.1) that $\mathscr{J}_{\mathcal{M}(\mathscr{E}(\pi))} \cap \mathscr{J}_{>w} \subseteq J(\mathscr{K})$. Recall that $\mathscr{J}_{\geq w} / \mathscr{J}_{>w} \simeq \sigma\left[\lambda_{\psi_{\pi}(w)}\right]$. Therefore, if $w \in \Omega_{\pi}$ then $\mathscr{J}_{\mathcal{M}(\mathscr{E}(\pi))} \cap \mathscr{J}_{>w}=\mathscr{J}_{\mathcal{M}(\mathscr{E}(\pi))} \cap \mathscr{J}_{\geq w}$ and the induction step follows trivially. On the other hand, if $w \notin \Omega_{\pi}$ then there exists $k$ such that $w=w_{1} w_{2}$ where $w_{1} \in \omega_{k}$ and $w_{2} \in \Omega_{M_{k}}$. By (4.3) and Corollary 3 applied with $L=M, M=M_{k}, \varrho=\delta$ and $\beta=\varrho_{k}$ we infer that

Hence

$$
J\left(\mathscr{K}_{k}\right)+\mathscr{J}_{>w}=J\left(I_{M_{k}}\left(\varrho_{k}\right)\right)+\mathscr{J}_{>w} \supseteq \mathscr{J}_{\geq w} .
$$

$$
\mathscr{J}_{\geq w} \cap \mathscr{J}_{\mathcal{M}(\mathscr{E}(\pi))} \subseteq J\left(\mathscr{K}_{k}\right) \cap \mathscr{J}_{\mathcal{M}(\mathscr{E}(\pi))}+\mathscr{J}_{>w} \cap \mathscr{J}_{\mathcal{M}(\mathscr{E}(\pi))} \subseteq J(\mathscr{K})+\mathscr{J}_{>w} \cap \mathscr{J}_{\mathcal{M}(\mathscr{E}(\pi))}
$$


and we can apply the induction hypothesis to conclude that $\mathscr{J}_{\geq w} \cap \mathscr{J}_{\mathcal{M}(\mathscr{E}(\pi))} \subseteq J(\mathscr{K})$ as required.

It remains to show the second part of Theorem 7.

Let $\mathfrak{M}: \Delta_{1} \times \cdots \times \Delta_{t} \rightarrow \Delta_{t} \times \cdots \times \Delta_{1}$ be the "longest" intertwining operator. It is known that the image of $\mathfrak{M}$ is $\pi$ (or equivalently, Ker $\mathfrak{M}=\mathscr{L}$ ). Since $J$ is a functor we have a map

$$
J(\mathfrak{M}): \mathscr{J} \rightarrow J\left(\Delta_{t} \times \cdots \times \Delta_{1}\right) .
$$

Lemma 9. $J(\mathfrak{M})$ factors through an injective map on $\mathscr{J}^{\mathcal{M}(\mathscr{E}(\pi))}$. Therefore $J(\pi) \simeq$ $\mathscr{J}^{\mathcal{M}(\mathscr{E}(\pi))}$.

Proof. It is clear that for each $k=1, \ldots, t-1, \mathfrak{M}$ factors through the intertwining operator obtained by switching $\Delta_{k-1}$ and $\Delta_{k}$. Hence, Ker $\mathfrak{M} \supseteq \mathscr{K}_{k}$ for all $k$ and consequently $\operatorname{Ker} \mathfrak{M} \supseteq \mathscr{K}$. Thus, Ker $J(\mathfrak{M}) \supseteq J(\mathscr{K})$ and by the first part of the Theorem proved above it follows that $J(\mathfrak{M})$ factors through $\mathscr{J}^{\mathcal{M}(\mathscr{E}(\pi))}$. It remains to show that $J(\mathfrak{M})$ is injective on $I=\mathscr{J}_{\mathcal{M}(\mathscr{G}) \backslash \mathcal{M}(\mathscr{E}(\pi))}$. Let $s_{i_{1}} \ldots s_{i_{l}}$ be a reduced decomposition of the longest element in $S_{t}$. Correspondingly we decompose

$$
\mathfrak{M}=\mathfrak{M}_{l} \circ \cdots \circ \mathfrak{M}_{1}
$$

where $\mathfrak{M}_{j}$ is the intertwining operator

$$
\mathfrak{M}_{j}: \Delta_{w_{j-1}(1)} \times \cdots \times \Delta_{w_{j-1}(t)} \rightarrow \Delta_{w_{j}(1)} \times \cdots \times \Delta_{w_{j}(t)}
$$

where $w_{j}=s_{i_{1}} \ldots s_{i_{j}}$. We will show that for all $j=1, \ldots, l J\left(\mathfrak{M}_{j}\right)$ is injective on the image of $I$ under $J\left(\mathfrak{M}_{j-1} \circ \cdots \circ \mathfrak{M}_{1}\right)$, i.e.

$$
\text { Ker } J\left(\mathfrak{M}_{j}\right) \cap J\left(\mathfrak{M}_{j-1} \circ \cdots \circ \mathfrak{M}_{1}\right)(I)=0 \text {. }
$$

Recall that $w_{j-1}\left(i_{j}\right)<w_{j-1}\left(i_{j}+1\right)=w_{j}\left(i_{j}\right)$ and $\operatorname{Ker} \mathfrak{M}_{j}$ is equivalent to $\Delta_{w_{j}(1)} \times \cdots \times \Delta_{w_{j}\left(i_{j}-1\right)} \times \Delta\left(\left[a_{w_{j-1}\left(i_{j}\right)}, b_{w_{j}\left(i_{j}\right)}\right]\right) \times \Delta\left(\left[a_{w_{j}\left(i_{j}\right)}, b_{w_{j-1}\left(i_{j}\right)}\right]\right) \times \Delta_{w_{j}\left(i_{j}+2\right)} \times \cdots \times \Delta_{w_{j}(t)}$ which is equal to $\mathcal{I}_{\left(w_{j-1}\left(i_{j}\right), w_{j}\left(i_{j}\right)\right)}$ in $\mathscr{R}$. It follows from (4.2) that $\left[\operatorname{Ker} \mathfrak{M}_{j}\right] \leq[\mathscr{K}]$, and hence $\left[\operatorname{Ker} J\left(\mathfrak{M}_{j}\right)\right]=\mathbf{J}\left(\operatorname{Ker} \mathfrak{M}_{j}\right) \leq \mathbf{J}(\mathscr{K})$. Since $[I]$ and $\mathbf{J}(\mathscr{K})$ are disjoint, we deduce (4.4).

This concludes the proof of Theorem 7.

\section{Application to TAdić's formula}

We continue to use the notation and assumptions of the previous section. Our goal in this section is to prove the second part of Theorem 1, namely Tadić's determinantal formula (1.1)

$$
\pi=\sum_{w \in S_{t}} \operatorname{sgn} w \mathcal{I}_{w}
$$

Let

$$
S_{t}^{\circ}:=\left\{w \in S_{t}: a_{w(i)} \leq b_{i}+1, i=1, \ldots, t\right\} .
$$

We note that only elements of $S_{t}^{\circ}$ contribute to the sum on the right-hand side of (1.1). 
Given $w \in S_{t}$ we have $w \in S_{t}^{\circ}$ if and only if $w(i) \geq r_{i}$ for all $i$ where $r_{i}=\min \left\{j: a_{j} \leq\right.$ $\left.\left.b_{i}+1\right\}\right) \leq i$. Thus $\left|S_{t}^{\circ}\right|=\prod_{i=1}^{t}\left(i+1-r_{i}\right)$. Indeed, $r_{1} \leq \cdots \leq r_{t}$; there are $t+1-r_{t}$ possibilities for $w(t), t-r_{t-1}$ possibilities for $w(t-1)$ given $w(t)$, etc.

Remark 4. In light of Remark 3 we can view (1.1) as a $p$-adic analogue of the Jacobi-Trudi identity.

5.1. To simplify notations, let $\mathscr{E}=\mathscr{E}\left(\mathrm{L}\left(\Delta_{1}, \ldots, \Delta_{t}\right)\right)$ and, for any $w \in S_{t}^{0}$, let $\mathscr{I}_{w}=$ $\mathscr{I}\left(\mathrm{L}\left(\Delta\left(\left[a_{w(1)}, b_{1}\right]\right), \ldots, \Delta\left(\left[a_{w(t)}, b_{t}\right]\right)\right)\right)$. Recall that $\mathcal{M}(\mathscr{E}) \subseteq \mathcal{M}\left(\mathscr{I}_{\mathrm{Id}}\right)$.

Set

$$
\mathcal{M}_{0}=\left\{(\phi, w): w \in S_{t}^{\circ}, \phi \in \mathcal{M}\left(\mathscr{I}_{w}\right)\right\} \backslash\{(\phi, \mathrm{Id}): \phi \in \mathcal{M}(\mathscr{E})\} .
$$

Proposition 10. There exists an involution $*$ on $\mathcal{M}_{0}$ such that if $(\phi, w)^{*}=\left(\phi^{*}, w^{*}\right)$ then $\operatorname{sgn}\left(w^{*}\right)=-\operatorname{sgn}(w)$ and $\lambda_{\phi}=\lambda_{\phi^{*}}$.

(In the case $t=2$ this involution was used in section 4.3 above.)

Before proving the proposition, it will be convenient to introduce the following convention. Given a graph $\mathscr{I}$ and $\phi \in \mathcal{M}(\mathscr{I})$ we extend $\phi$ to $\mathbb{Z}^{2}$ by setting

$$
\phi(j, i)= \begin{cases}\infty & j<a_{i}, i=1, \ldots, t, \\ -\infty & j>b_{i}, i=1, \ldots, t, \\ \infty & i<1, \\ -\infty & i>t .\end{cases}
$$

Example 4. In example 3, $\phi_{\text {std }}$ extends to

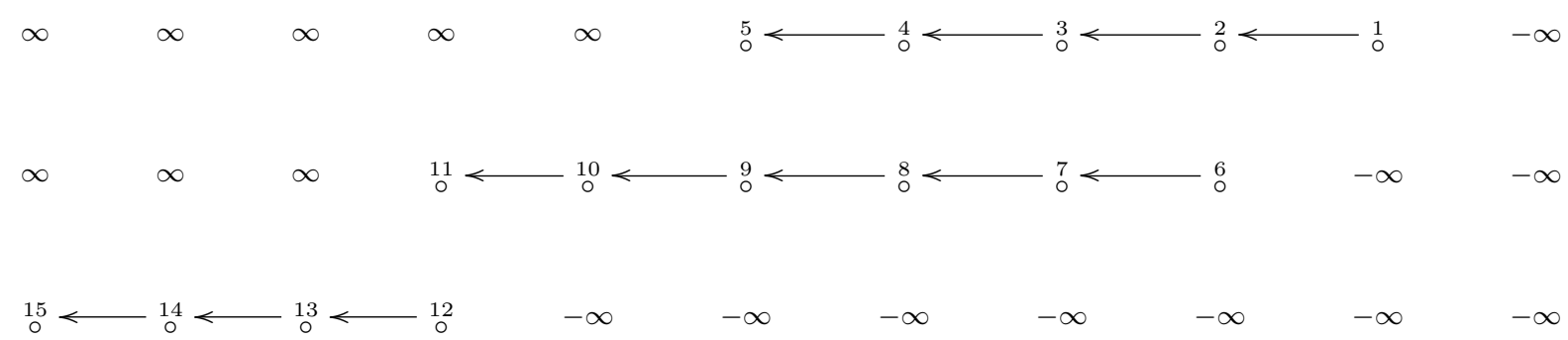

Proof. The statement and the proof are closely related to the Gessel-Viennot Lemma (cf. [Sta97, Theorem 2.7.1]). Let $(\phi, w) \in \mathcal{M}_{0}$. Define

$$
Y=Y_{(\phi, w)}=\left\{(j, i) \in \mathbb{Z}^{2}: \phi(j, i)<\phi(j-1, i+1)\right\} .
$$

Note that by our convention we have $\left(a_{w(i+1)}, i\right) \in Y$ whenever $w(i)>w(i+1)$. Thus, $Y \neq$ $\emptyset$. Also, we have $1 \leq i<t$ and $j \in\left[a_{w(i)}, b_{i+1}+1\right]$ for all $(j, i) \in Y$. Let $\left(j_{0}, i_{0}\right)=\left(j_{0}^{\phi}, i_{0}^{\phi}\right)$ be the maximal element in $Y$ with respect to the lexicographic order (from left to right). Clearly $j_{0} \in\left[a_{\min \left(w\left(i_{0}\right), w\left(i_{0}+1\right)\right)}, b_{i_{0}+1}+1\right]$.

Let $s_{i_{0}}$ be the transposition $\left(i_{0}, i_{0}+1\right)$, set $w^{*}=w s_{i_{0}}$ and define

$$
\phi^{*}(j, i)= \begin{cases}\phi\left(j, s_{i_{0}}(i)\right) & j<j_{0}, \\ \phi(j, i) & \text { otherwise. }\end{cases}
$$


By definition, it is clear that $\lambda_{\phi^{*}}=\lambda_{\phi}$ and $\operatorname{sgn}\left(w^{*}\right)=-\operatorname{sgn}(w)$. Let us check that $w^{*} \in S_{t}^{0}$. It is clear that $a_{w^{*}(i)} \leq b_{i}+1$ for $i \neq i_{0}, i_{0}+1$. Also

$$
a_{w^{*}\left(i_{0}\right)}=a_{w\left(i_{0}+1\right)} \leq j_{0} \leq b_{i_{0}+1}+1<b_{i_{0}}+1
$$

and

$$
a_{w^{*}\left(i_{0}+1\right)}=a_{w\left(i_{0}\right)} \leq j_{0} \leq b_{i_{0}+1}+1
$$

Thus, $w^{*} \in S_{t}^{0}$.

Next, we show that $\phi^{*} \in \mathcal{M}\left(\mathscr{I}_{w^{*}}\right)$. It is clear that for all $1 \leq i \leq t$ we have $\phi^{*}(j, i)=\infty$ if and only if $j<a_{w^{*}(i)}$ and $\phi^{*}(j, i)=-\infty$ if and only if $j>b_{i}$. We also need to show that for all $1 \leq i \leq t$ and $j \in\left[a_{w^{*}(i)}+1, b_{i}\right]$ we have $\phi^{*}(j-1, i)>\phi^{*}(j, i)$. This follows from the similar property of $\phi$ if either $i \neq i_{0}, i_{0}+1$ or $j \neq j_{0}$. For the remaining two cases we have

$$
\phi^{*}\left(j_{0}-1, i_{0}\right)=\phi\left(j_{0}-1, i_{0}+1\right)>\phi\left(j_{0}, i_{0}\right)=\phi^{*}\left(j_{0}, i_{0}\right)
$$

since $\left(j_{0}, i_{0}\right) \in Y$, and

$$
\phi^{*}\left(j_{0}-1, i_{0}+1\right)=\phi\left(j_{0}-1, i_{0}\right)>\phi\left(j_{0}, i_{0}\right)>\phi\left(j_{0}+1, i_{0}\right)>\phi\left(j_{0}, i_{0}+1\right)=\phi^{*}\left(j_{0}, i_{0}+1\right)
$$

where the last inequality follows from the maximality of $\left(j_{0}, i_{0}\right)$.

Finally we verify that $\left(\phi^{*}, w^{*}\right)^{*}=(\phi, w)$. Let $\left(j_{0}, i_{0}\right)=\left(j_{0}^{\phi}, i_{0}^{\phi}\right),\left(j_{0}^{*}, i_{0}^{*}\right)=\left(j_{0}^{\phi^{*}}, i_{0}^{\phi^{*}}\right), Y=$ $Y_{(\phi, w)}$ and $Y^{*}=Y_{\left(\phi^{*}, w^{*}\right)}$. Observe that $\phi^{*}\left(j_{0}, i_{0}\right)=\phi\left(j_{0}, i_{0}\right)<\phi\left(j_{0}-1, i_{0}\right)=\phi^{*}\left(j_{0}-1, i_{0}+1\right)$, so that $\left(j_{0}, i_{0}\right) \in Y^{*}$. Suppose that $(j, i)>\left(j_{0}, i_{0}\right)$ in the lexicographic order. Then $\phi^{*}(j, i)=\phi(j, i)$ (since $\left.j \geq j_{0}\right)$ and $\phi^{*}(j-1, i+1)=\phi(j-1, i+1)\left(\right.$ since $j \geq j_{0}$ and if $j=j_{0}$ then $\left.i>i_{0}\right)$. Thus, $\phi^{*}(j, i)>\phi^{*}(j-1, i+1)$ and $(j, i) \notin Y^{*}$. Thus, $\left(j_{0}, i_{0}\right)$ is the maximal element in $Y^{*}$, so that $\left(j_{0}^{*}, i_{0}^{*}\right)=\left(j_{0}, i_{0}\right)$. It readily follows that $\left(\phi^{*}, w^{*}\right)^{*}=(\phi, w)$.

Corollary 11. We have an equality in the Grothendieck group:

$$
\mathbf{X}_{\mathcal{M}\left(\mathscr{E}\left(\mathrm{L}\left(\Delta_{1}, \ldots, \Delta_{t}\right)\right)\right)}=\sum_{w \in S_{t}} \operatorname{sgn}(w) \mathbf{X}_{\mathcal{M}\left(\mathscr{I}_{w}\right)}
$$

Equivalently (by (3.2)),

$$
\mathbf{J}\left(\mathrm{L}\left(\Delta_{1}, \ldots, \Delta_{t}\right)\right)=\sum_{w \in S_{t}} \operatorname{sgn}(w) \mathbf{J}\left(\mathcal{I}_{w}\right)
$$

Proof. First note that only $w \in S_{t}^{0}$ contributes. With the notation as in the previous proposition, we have

$$
\begin{aligned}
\sum_{w \in S_{t}^{0}} \operatorname{sgn}(w) \mathbf{X}_{\mathcal{M}\left(\mathscr{I}_{w}\right)} & =\sum_{w \in S_{t}^{0}} \operatorname{sgn}(w) \sum_{\phi \in \mathcal{M}\left(\mathscr{I}_{w}\right)} \sigma\left[\lambda_{\phi}\right] \\
& =\mathbf{X}_{\mathcal{M}(\mathscr{E})}+\sum_{(\phi, w) \in \mathcal{M}_{0}} \operatorname{sgn}(w) \sigma\left[\lambda_{\phi}\right]
\end{aligned}
$$

But, applying the involution, we have $\sum_{(\phi, w) \in \mathcal{M}_{0}} \operatorname{sgn}(w) \sigma\left[\lambda_{\phi}\right]=0$. The corollary follows. 
5.2. Proof of (1.1). It is known that

$$
\mathrm{L}\left(\Delta_{1}, \ldots, \Delta_{t}\right)=\sum_{w \in S_{t}^{\circ}} c_{w} \mathcal{I}_{w}
$$

for some integers $c_{w}$. Applying $\mathbf{J}$ we obtain

$$
\mathbf{J}\left(\mathrm{L}\left(\Delta_{1}, \ldots, \Delta_{t}\right)\right)=\sum_{w \in S_{t}^{\circ}} c_{w} \mathbf{J}\left(\mathcal{I}_{w}\right) .
$$

Combined with (5.2), we infer that

$$
\sum_{w \in S_{t}^{\circ}} \operatorname{sgn}(w) \mathbf{J}\left(\mathcal{I}_{w}\right)=\sum_{w \in S_{t}^{\circ}} c_{w} \mathbf{J}\left(\mathcal{I}_{w}\right) .
$$

On the other hand by [Zel80, $\S 6.9]$, the elements $\mathbf{J}\left(\mathcal{I}_{w}\right), w \in S_{t}^{\circ}$ are free over $\mathbb{Z}$ in $\mathscr{R}$. We conclude that $c_{w}=\operatorname{sgn}(w)$ for all $w$. This concludes the proof of Theorem 1 .

5.3. Note that if we define the matrix $\left(m_{i, j}\right)_{i, j=1, \ldots, t}$ with coefficients in $\mathscr{R}$ by $m_{i, j}=$ $\Delta\left(\left[a_{i}, b_{j}\right]\right)$ then the identity $(1.1)$ reads

$$
\mathrm{L}\left(\Delta_{1}, \ldots, \Delta_{t}\right)=\operatorname{det}\left(m_{i, j}\right) .
$$

Thus, by [CR08, Lemma 6.1], we immediately get the following result.

Corollary 12. Let $\Delta_{i}=\Delta\left(\left[a_{i}, b_{i}\right]\right), i=1, \ldots, t$ be a ladder. Let

$$
\begin{aligned}
\pi & =\mathrm{L}\left(\Delta_{1}, \ldots, \Delta_{t-1}\right) \times \mathrm{L}\left(\Delta_{2}, \ldots, \Delta_{t}\right), \\
\pi_{1} & =\mathrm{L}\left(\Delta_{1}, \ldots, \Delta_{t}\right) \times \mathrm{L}\left(\Delta_{2}, \ldots, \Delta_{t-1}\right), \\
\pi_{2} & =\mathrm{L}\left(\Delta\left(\left[a_{1}, b_{2}\right]\right), \ldots, \Delta\left(\left[a_{t-1}, b_{t}\right]\right)\right) \times \mathrm{L}\left(\Delta\left(\left[a_{2}, b_{1}\right]\right), \ldots, \Delta\left(\left[a_{t}, b_{t-1}\right]\right)\right) .
\end{aligned}
$$

Then in the Grothendieck group we have $\pi=\pi_{1}+\pi_{2}$.

For Speh representations see [Tad06].

Remark 5. Note that $\pi_{2}=0$ if $a_{i}>b_{i+1}+1$ for some $i=1, \ldots, t-1$. In a subsequent paper $[\mathrm{BLM}]$ we show that $\pi=\pi_{1}$ is irreducible in this case. Otherwise we show that $\pi_{1}$ and $\pi_{2}$ are irreducible, so that $\pi$ has length 2 , and $\pi_{1}$ (resp. $\pi_{2}$ ) is the unique irreducible quotient (resp. subrepresentation) of $\pi$.

5.4. We can rephrase Theorem 1 in terms of Zelevinsky classification [Zel80]. For any segment $[a, b]$ we write $Z([a, b])=\mathrm{L}\left(\sigma \nu^{b}, \ldots, \sigma \nu^{a}\right)$. Thus $Z([a, b])$ is the unique irreducible subrepresentation of $\sigma \nu^{a} \times \cdots \times \sigma \nu^{b}$. As usual we write $Z([a, a-1])=1$ and $Z([a, b])=0$ if $b<a-1$. More generally, for a multisegment $\left[a_{1}, b_{1}\right], \ldots,\left[a_{t}, b_{t}\right]$ such that for any $i<j$ $\left[a_{i}, b_{i}\right]$ does not precede $\left[a_{j}, b_{j}\right]$ we write $Z\left(\left[a_{1}, b_{1}\right], \ldots,\left[a_{t}, b_{t}\right]\right)$ for the unique irreducible subrepresentation of $Z\left(\left[a_{1}, b_{1}\right]\right) \times \cdots \times Z\left(\left[a_{t}, b_{t}\right]\right)$.

The Zelevinsky involution takes $L\left(\Delta\left(\left[a_{1}, b_{1}\right]\right), \ldots, \Delta\left(\left[a_{t}, b_{t}\right]\right)\right)$ to $Z\left(\left[a_{1}, b_{1}\right], \ldots,\left[b_{t}, a_{t}\right]\right)$.

Recall that the class of ladder representations is invariant under Zelevinsky involution. Therefore, if $a_{1}>\cdots>a_{t}$ and $b_{1}>\cdots>b_{t}$ then $Z\left(\left[a_{1}, b_{1}\right], \ldots,\left[a_{t}, b_{t}\right]\right)$ is a ladder representation and conversely any ladder representation can be expressed this way.

By applying Zelevinsky involution on (1.1) we obtain 
Corollary 13. Suppose that $a_{1}>\cdots>a_{t}$ and $b_{1}>\cdots>b_{t}$. Then we have

$$
\begin{array}{r}
Z\left(\left[a_{1}, b_{1}\right]\right) \times \cdots \times Z\left(\left[a_{t}, b_{t}\right]\right)=\sum_{w \in S_{t}^{\circ}} \operatorname{sgn} w Z\left(\left[a_{w(1)}, b_{1}\right]\right) \times \cdots \times Z\left(\left[a_{w(t)}, b_{t}\right]\right) \\
=\operatorname{det}\left(Z\left(\left[a_{i}, b_{j}\right]\right)\right)_{1 \leq i, j \leq t}
\end{array}
$$

in $\mathscr{R}$.

5.5. We conclude this section with another application, due to Marko Tadić, for the computation of the (full) derivative of a ladder representation. This formula had been conjectured by Tadić for Speh representations [Tad87]. The interesting point is that even for Speh representations one needs to use non-unitary ladder representations. We are grateful to Marko Tadić for kindly allowing us to include this application here.

Recall that for any finite length representation $\pi$ of $G_{n}$ we can consider its full derivative $\mathcal{D}(\pi)$ which is a sequence of finite length representations of $G_{i}, i=0, \ldots, n$ (see [BZ77, §4] for definition and basic properties). The functor $\mathcal{D}$ on $\oplus_{n=0}^{\infty} \mathscr{C}^{G_{n}}$ induces a ring homomorphism of the Grothendieck group (which is a subring of $\mathscr{R}$ ). We have $\mathcal{D}(Z([a, b]))=Z([a, b])+Z([a, b-1])([$ Zel80, Theorem 3.5]).

Theorem 14 (Tadić). Suppose that $a_{1}>\cdots>a_{t}$ and $b_{1}>\cdots>b_{t}$. Then the full derivative $\mathcal{D}\left(Z\left(\left[a_{1}, b_{1}\right], \ldots,\left[a_{t}, b_{t}\right]\right)\right)$ of $Z\left(\left[a_{1}, b_{1}\right], \ldots,\left[a_{t}, b_{t}\right]\right)$ is given by the direct sum of

$$
Z\left(\left[a_{1}, b_{1}^{\prime}\right], \ldots,\left[a_{t}, b_{t}^{\prime}\right]\right)
$$

where $\left(b_{1}^{\prime}, \ldots, b_{t}^{\prime}\right)$ range over all sequences such that $b_{i}-b_{i}^{\prime} \in\{0,1\}$ for all $i$ and $b_{1}^{\prime}<\cdots<$ $b_{t}^{\prime}$. In other words,

$$
\mathcal{D}\left(Z\left(\left[a_{1}, b_{1}\right], \ldots,\left[a_{t}, b_{t}\right]\right)\right)=\oplus_{\mathfrak{m}^{\prime}} Z\left(\mathfrak{m}^{\prime}\right)
$$

where the sum is over all multisegments $\mathfrak{m}^{\prime}$ which are subordinate to $\left[a_{1}, b_{1}\right], \ldots,\left[a_{n}, b_{n}\right]$ (in the sense of [Zel80, §7.4]) and which form a ladder.

Proof. Using Corollary 13 and the fact that $\mathcal{D}$ is a ring homomorphism we have

$$
\begin{aligned}
\mathcal{D}\left(Z\left(\left[a_{1}, b_{1}\right], \ldots,\left[a_{t}, b_{t}\right]\right)\right)=\mathcal{D}\left(\operatorname{det}\left(Z\left(\left[a_{i}, b_{j}\right]\right)\right)_{1 \leq i, j \leq t}\right)=\operatorname{det}\left(\mathcal{D}\left(Z\left(\left[a_{i}, b_{j}\right]\right)\right)\right)_{1 \leq i, j \leq t} \\
=\operatorname{det}\left(Z\left(\left[a_{i}, b_{j}\right]\right)+Z\left(\left[a_{i}, b_{j}-1\right]\right)\right)_{1 \leq i, j \leq t}
\end{aligned}
$$

in $\mathscr{R}$. By the multi-linearity of the determinant we get

$$
\sum_{\epsilon_{1}, \ldots, \epsilon_{t} \in\{0,1\}} \operatorname{det}\left(Z\left(\left[a_{i}, b_{j}-\epsilon_{j}\right]\right)\right)_{1 \leq i, j \leq t} .
$$

Let $b_{j}^{\prime}=b_{j}-\epsilon_{j}$ so that $b_{j}^{\prime} \geq b_{j+1}^{\prime}, j=1, \ldots, t-1$. If $b_{j}^{\prime}=b_{j+1}^{\prime}$ for some $j$ then the corresponding determinant in the sum above vanishes, since the matrix contains two identical columns. Therefore we remain with

$$
\sum_{\forall j b_{j}-b_{j}^{\prime} \in\{0,1\}, b_{1}^{\prime}<\cdots<b_{t}^{\prime}} \operatorname{det}\left(Z\left(\left[a_{i}, b_{j}^{\prime}\right]\right)\right)_{1 \leq i, j \leq t} .
$$


Once again using Corollary 13 we obtain

$$
\mathcal{D}\left(Z\left(\left[a_{1}, b_{1}\right], \ldots,\left[a_{t}, b_{t}\right]\right)\right)=\sum_{\forall j b_{j}-b_{j}^{\prime} \in\{0,1\}, b_{1}^{\prime}<\cdots<b_{t}^{\prime}} Z\left(\left[a_{1}, b_{1}^{\prime}\right], \ldots,\left[a_{t}, b_{t}^{\prime}\right]\right)
$$

in $\mathscr{R}$. Now observe that the cuspidal supports of all representations in the above sum are different. Therefore, their infinitesimal characters (in the sense of Bernstein center) are all different. Hence,

$$
\mathcal{D}\left(Z\left(\left[a_{1}, b_{1}\right], \ldots,\left[a_{t}, b_{t}\right]\right)\right)=\underset{\forall j b_{j}-b_{j}^{\prime} \in\{0,1\}, b_{1}^{\prime}<\cdots<b_{t}^{\prime}}{ } Z\left(\left[a_{1}, b_{1}^{\prime}\right], \ldots,\left[a_{t}, b_{t}^{\prime}\right]\right)
$$

as an isomorphism of representations (of the various $\mathrm{GL}_{n}$ 's).

Note the similarity between this argument and the standard proof of branching laws for the unitary group or the symmetric group (e.g., [Bum04, ch. 42, 44]).

We can rephrase Theorem 14 in terms of Langlands classification as follows. If $\Delta_{1}, \ldots, \Delta_{t}$ form a ladder with $\Delta_{i}=\Delta\left(\left[a_{i}, b_{i}\right]\right)$ then

$$
\mathcal{D}\left(\mathrm{L}\left(\Delta_{1}, \ldots, \Delta_{t}\right)\right)=\sum \mathrm{L}\left(\Delta\left(\left[a_{1}^{\prime}, b_{1}\right]\right), \ldots, \Delta\left(\left[a_{t}^{\prime}, b_{t}\right]\right)\right)
$$

where the sum is over $a_{i}^{\prime} \in\left[a_{i}, a_{i-1}-1\right], i=1, \ldots, t$ with the convention that $a_{0}=\infty$. (In particular, $a_{1}^{\prime}>\cdots>a_{n}^{\prime}$.) This can be proved by applying the Mœglin-Waldspurger algorithm to both sides of (5.3). Alternatively, we can follow the argument of Theorem 14 using the fact that $\mathcal{D}([a, b])=\sum_{a^{\prime} \geq a} \Delta\left(\left[a^{\prime}, b\right]\right)$ ([Zel80, Proposition 9.6]) - of course only $a^{\prime} \leq b+1$ contribute. We will obtain

$$
\mathcal{D}\left(\mathrm{L}\left(\Delta_{1}, \ldots, \Delta_{t}\right)\right)=\sum_{a_{i}^{\prime} \geq a_{i}, i=1, \ldots, t} \operatorname{det} \Delta\left(\left[a_{i}^{\prime}, b_{j}\right]\right) .
$$

If the $a_{j}^{\prime}$ are not distinct then the corresponding summand vanishes. The summands for which $a_{i}^{\prime} \geq a_{i-1}$ for some $i>1$ cancel in pairs. Indeed, on this set we can define an involution by switching $a_{i_{0}}^{\prime}$ and $a_{i_{0}-1}^{\prime}$ where $i_{0}>1$ is the largest index such that $a_{i_{0}}^{\prime} \geq a_{i_{0}-1}^{\prime}$. This involution negates the corresponding summand. We remain with the $a_{i}^{\prime}$ such that $a_{i}^{\prime}<a_{i-1}$ as required.

\section{ODDS AND ENDS}

We will list several questions and conjectures arising from the results above.

6.1. Decomposition of standard modules. It is natural to ask whether in the case of a ladder representations $\mathrm{L}\left(\Delta_{1}, \ldots, \Delta_{t}\right)$ the decomposition of $\Delta_{1} \times \cdots \times \Delta_{t}$ in $\mathscr{R}$, or more generally of $M_{w}:=\mathcal{I}_{w}$ for any $w \in S_{t}^{\circ}$ (see (5.1)), is any simpler than in the general case.

As was pointed out by Tadić, $M_{\text {Id }}$ is not multiplicity free in general for $t>3$ [Tad95, $\S 6]$. Let $L_{w}=\mathrm{L}\left(\Delta\left(\left[a_{w(1)}, b_{1}\right]\right), \ldots, \Delta\left(\left[a_{w(t)}, b_{t}\right]\right)\right)$. Recall that $L_{w} \neq 0$ if and only if $w \in S_{t}^{\circ}$. Note that for $w, w^{\prime} \in S_{t}^{\circ}$ we have $w^{\prime} \geq w$ if and only if

$$
\left(\left[a_{w^{\prime}(1)}, b_{1}\right], \ldots,\left[a_{w^{\prime}(t)}, b_{t}\right]\right) \leq\left(\left[a_{w(1)}, b_{1}\right], \ldots,\left[a_{w(t)}, b_{t}\right]\right)
$$


in the partial order on multi-segments introduced by Zelevinsky in [Zel80, §7]. Therefore we have

$$
M_{w}=\sum_{w^{\prime} \geq w} c_{w, w^{\prime}} L_{w^{\prime}}
$$

for some coefficients $c_{w, w^{\prime}} \in \mathbb{Z}_{\geq 0}$. We have $c_{w, w}=1$ for all $w \in S_{t}^{\circ}$.

Lemma 15. There exists a unique $w_{0}^{\prime} \in S_{t}^{\circ}$ such that

$$
S_{t}^{\circ}=\left\{w \in S_{t}: w \leq w_{0}^{\prime}\right\} .
$$

Moreover, $w_{0}^{\prime}$ avoids 312 pattern, i.e. there does not exist a triple $i<j<k$ such that $w_{0}^{\prime}(k)<w_{0}^{\prime}(i)<w_{0}^{\prime}(j)$. Finally $L_{w_{0}^{\prime}}$ is generic.

Proof. We first note that the set $S_{t}^{\circ}$ is closed from below in $S_{t}$ (i.e., it is closed in the Bruhat order topology of $S_{t}$ - see $\left.\S 2.6\right)$. Indeed, suppose that $w \in S_{t}^{\circ}$ and $w(i)>w(j)$ for some $i<j$. Let $s$ be the transposition $(i, j)$. We verify that $w^{\prime}:=w s \in S_{t}^{\circ}$. If $k \neq i, j$ then $a_{w^{\prime}(k)}=a_{w(k)} \leq b_{k}+1$. On the other hand, $a_{w^{\prime}(i)}=a_{w(j)} \leq b_{j}+1<b_{i}+1$ and $a_{w^{\prime}(j)}=a_{w(i)}<a_{w(j)} \leq b_{j}+1$. Thus $w^{\prime} \in S_{t}^{\circ}$ and $S_{t}^{\circ}$ is closed from below as claimed.

It remains to show that $S_{t}^{\circ}$ has a unique maximal element $w_{0}^{\prime}$. We define $w_{0}^{\prime}$ by recursion as follows. Suppose that $w_{0}^{\prime}(t), \ldots, w_{0}^{\prime}(i+1)$ were defined for some $1 \leq i \leq t$. Then define $w_{0}^{\prime}(i)$ to be the minimal index $j \neq w_{0}^{\prime}(t), \ldots, w_{0}^{\prime}(i+1)$ such that $a_{j} \leq b_{i}+1$. (Such $j$ exists because $a_{i}, \ldots, a_{t} \leq b_{i}+1$.) By definition, $w_{0}^{\prime} \in S_{t}^{\circ}$. To see that $w_{0}^{\prime}$ is the unique maximal element of $S_{t}^{\circ}$ suppose that $w \in S_{t}^{\circ}$ with $w \neq w_{0}^{\prime}$. Then we claim that $w$ is not maximal in $S_{t}^{\circ}$. Indeed, let $i$ be the maximal index such that $w(i) \neq w_{0}^{\prime}(i)$. Then by definition of $w_{0}^{\prime}$ we necessarily have $w(i)>w_{0}^{\prime}(i)$. Let $s$ be the transposition $(i, j)$ with $j=w^{-1}\left(w_{0}^{\prime}(i)\right)$. Note that $j<i$ since $w(k)=w^{\prime}(k)$ for all $k>i$ by assumption. Also, $w(j)=w_{0}^{\prime}(i)<w(i)$ so that $w s>w$. On the other hand $w s \in S_{t}^{\circ}$ because $a_{w s(k)}=a_{w(k)} \leq b_{k}+1$ for all $k \neq i, j$ while $a_{w s(i)}=a_{w(j)}=a_{w_{0}^{\prime}(i)} \leq b_{i}+1$ and $a_{w s(j)}=a_{w(i)} \leq b_{i}+1 \leq b_{j}+1$.

We conclude (6.1).

Let us prove now that $w_{0}^{\prime}$ avoids the pattern 312. Assume on the contrary that it does not. Then $w_{0}^{\prime}(k)<w_{0}^{\prime}(i)<w_{0}^{\prime}(j)$ for some triple $i<j<k$. It follows that $a_{w_{0}^{\prime}(i)}<a_{w_{0}^{\prime}(k)} \leq b_{k}+1<b_{j}+1$. However, by the choice of $w_{0}^{\prime}(j)$ we would then have $w_{0}^{\prime}(j) \leq w_{0}^{\prime}(i)$ in contradiction.

Finally, to show that $L_{w_{0}^{\prime}}$ is generic assume on the contrary that $\left[a_{w_{0}^{\prime}(j)}, b_{j}\right]$ precedes $\left[a_{w_{0}^{\prime}(i)}, b_{i}\right]$ for some $i<j$. Then $w_{0}^{\prime}(i)<w_{0}^{\prime}(j)$ and $a_{w_{0}^{\prime}(i)} \leq b_{j}+1$ in contradiction with the minimality of $w_{0}^{\prime}(j)$.

Remark 6. One can show conversely that any $w \in S_{t}$ which avoids 312 pattern can be realized as $w_{0}^{\prime}$ for some ladder of rank $t$. These permutations are exactly the inverses of the stack sortable permutations. Their number is known to be the Catalan number $C_{t}$ ([Sta99, p. 224]).

We conclude that $c_{w, w^{\prime}}>0$ for all $w \leq w^{\prime} \leq w_{0}^{\prime}$ and $c_{w, w_{0}^{\prime}}=1$ for all $w \in S_{t}^{\circ}$. Set $c_{w, w^{\prime}}=0$ if $w \not \leq w^{\prime}$. Let $c_{w, w^{\prime}}^{\prime}$ be the inverse matrix of $\left(c_{w, w^{\prime}}\right)_{w, w^{\prime} \in S_{t}^{\circ}}$. Then $c_{w, w^{\prime}}^{\prime}$ has integer 
entries, $c_{w, w}^{\prime}=1$ for all $w \in S_{t}^{\circ}$ and $c_{w, w^{\prime}}^{\prime}=0$ if $w \not \leq w^{\prime}$. We have

$$
L_{w}=\sum_{w^{\prime} \geq w} c_{w, w^{\prime}}^{\prime} M_{w^{\prime}}
$$

Conjecture 1. We have $c_{w, w^{\prime}}=P_{w, w^{\prime}}(1)$ where $P_{w, w^{\prime}}$ are the Kazhdan-Lusztig polynomials. Equivalently,

$$
c_{w, w^{\prime}}^{\prime}=(-1)^{l(w)-l\left(w^{\prime}\right)} P_{w_{0} w^{\prime}, w_{0} w}(1) .
$$

In other words, the relation between the $M_{w}$ 's and $L_{w}$ 's is analogous to the relation between Verma modules and simple highest weight modules in the category $\mathscr{O}(c f$. [Hum08]).

Note that the relation (1.1) is the case $w=\operatorname{Id}$ of (6.2). Also, note that $P_{w, w_{0}^{\prime}} \equiv 1$ for all $w \leq w_{0}^{\prime}$ because $w_{0}^{\prime}$ avoids the pattern 312 [LS90]. This is consistent with the fact that $c_{w, w_{0}^{\prime}}=1$.

In principle, it should be possible to check whether Conjecture 1 is in accordance with Zelevinsky's conjectures [Zel85] proved in [CG97]. However, this is not straightforward since the Kazhdan-Lusztig polynomials appearing in [Zel85] are pertaining to the much bigger symmetric group $S_{m}$ where we recall that $m=\sum_{i=1}^{t}\left(b_{i}+1-a_{i}\right)$. We will not pursue this question any further here. At any rate, we checked that Conjecture 1 holds for $t=3$, in which case $c_{w, w^{\prime}}=1$ for all $w \leq w^{\prime}$, and $t=4$, in which case

$$
c_{w, w^{\prime}}= \begin{cases}0 & w^{\prime} \geq w, \\ 2 & w^{\prime}=(1,3)(2,4) \text { and } w \leq(2,3), \\ 2 & w^{\prime}=(1,4) \text { and } w \leq(1,2)(3,4), \\ 1 & \text { otherwise. }\end{cases}
$$

(See $[\operatorname{Tad} 95, \S 6]$ for a special case.)

Another interesting problem in connection with ladder representations would be to determine (the semisimplification of) all Jacquet modules, not only the minimal one. This seems to be unknown even for Speh representations. As was pointed out to us by Arno Kret, knowing it in this case would already have interesting consequences.

6.2. Imprimitive representations. We say that $\pi \in \operatorname{Irr} G_{n}$ is (parabolically) imprimitive if it is not (fully) induced from a proper parabolic subgroup. It is known that any $\pi \in \operatorname{Irr}$ can be expressed as the product of imprimitive representations in a unique way, up to reordering. In other words, the imprimitive representations are, roughly speaking, the prime elements of Irr. Thus, it is desirable to characterize imprimitive representations in terms of their Zelevinsky (or Langlands) data.

In general, by [Zel80, Proposition 8.4], if $\mathrm{L}\left(\Delta_{1}, \ldots, \Delta_{t}\right)$ is not imprimitive then there exists a non-trivial partition $\left\{i_{1}, \ldots, i_{r}\right\} \sqcup\left\{j_{1}, \ldots, j_{t-r}\right\}=\{1, \ldots, t\}$, such that

$$
\mathrm{L}\left(\Delta_{1}, \ldots, \Delta_{t}\right) \simeq \mathrm{L}\left(\Delta_{i_{1}}, \ldots, \Delta_{i_{r}}\right) \times \mathrm{L}\left(\Delta_{j_{1}}, \ldots, \Delta_{j_{t-r}}\right) .
$$

In the case of ladder representations we can easily describe the imprimitive ones. More precisely, we say that a ladder is proper if $a_{i} \leq b_{i+1}+1$ (i.e., $\Delta_{i+1}$ precedes $\Delta_{i}$ ) for all $i=1, \ldots, t-1$. Then we have 
Theorem 16. A ladder is proper if and only if $\mathrm{L}\left(\Delta_{1}, \ldots, \Delta_{t}\right)$ is imprimitive.

Proof. Suppose that $a_{i}>b_{i+1}+1$ for some $i$. Then it follows from [Zel80, Proposition 8.5] that

$$
\mathrm{L}\left(\Delta_{1}, \ldots, \Delta_{t}\right) \simeq \mathrm{L}\left(\Delta_{1}, \ldots, \Delta_{i}\right) \times \mathrm{L}\left(\Delta_{i+1}, \ldots, \Delta_{t}\right) .
$$

Hence $\mathrm{L}\left(\Delta_{1}, \ldots, \Delta_{t}\right)$ is not imprimitive.

Conversely, assume on the contrary that $\Delta_{1}, \ldots, \Delta_{t}$ is a proper ladder but $\mathrm{L}\left(\Delta_{1}, \ldots, \Delta_{t}\right)$ is not imprimitive. Then we have (6.3) for some non-trivial partition. By (1.1) (applied to the ladders $\left(\Delta_{1}, \ldots, \Delta_{t}\right),\left(\Delta_{i_{1}}, \ldots, \Delta_{i_{r}}\right)$ and $\left.\left(\Delta_{j_{1}}, \ldots, \Delta_{j_{t-r}}\right)\right)$ and the geometric Lemma we would obtain

$$
\sum_{w \in S_{t}^{\circ}} \operatorname{sgn} w \mathcal{I}_{w}=\sum_{w \in B} \operatorname{sgn} w \mathcal{I}_{w}
$$

where

$$
B=\left\{w \in S_{t}^{\circ}:\left\{w\left(i_{1}\right), \ldots, w\left(i_{r}\right)\right\}=\left\{i_{1}, \ldots, i_{r}\right\}\right\} .
$$

Since $\mathcal{I}_{w}, w \in S_{t}^{\circ}$ are free over $\mathbb{Z}$ in $\mathscr{R}$ this would mean that $B=S_{t}^{\circ}$. However, since $\Delta_{1}, \ldots, \Delta_{t}$ is a proper ladder, the transpositions $s_{i}, i=1, \ldots, t-1$ are in $S_{t}^{\circ}$. This leads to a contradiction.

We remark that one can easily give examples of non-ladder imprimitive representations. For instance, consider $t=3$ and a ladder $\left[a_{1}, b_{1}\right],\left[a_{2}, b_{2}\right],\left[a_{3}, b_{3}\right]$ such that $a_{1} \leq b_{3}+1$ (i.e., $w_{0}^{\prime}=(1,3)$ is the longest element of $S_{3}$ ). (The smallest such example is $[2,3]$, $[1,2],[0,1]$.) We claim that in the notation of the previous section, the representation $L_{(2,3)}$ (the Langlands quotient $\left.\mathrm{L}\left(\Delta\left(\left[a_{1}, b_{1}\right]\right), \Delta\left(\left[a_{3}, b_{2}\right]\right), \Delta\left(\left[a_{2}, b_{3}\right]\right)\right)\right)$ is imprimitive. Indeed, otherwise it would equal to one of the representations $\Delta\left(\left[a_{1}, b_{1}\right]\right) \times \mathrm{L}\left(\Delta\left(\left[a_{3}, b_{2}\right]\right), \Delta\left(\left[a_{2}, b_{3}\right]\right)\right)$, $\Delta\left(\left[a_{3}, b_{2}\right]\right) \times \mathrm{L}\left(\Delta\left(\left[a_{1}, b_{1}\right]\right), \Delta\left(\left[a_{2}, b_{3}\right]\right)\right)$ or $\Delta\left(\left[a_{2}, b_{3}\right]\right) \times \mathrm{L}\left(\Delta\left(\left[a_{1}, b_{1}\right]\right), \Delta\left(\left[a_{3}, b_{2}\right]\right)\right)$. However, these representations are $M_{(2,3)}, M_{(2,3)}-M_{(1,2,3)}$ and $M_{(2,3)}-M_{(1,3,2)}$ respectively and none of them is irreducible since $L_{(2,3)}+L_{(1,3,2)} \leq M_{(2,3)}-M_{(1,2,3)}$ and $L_{(2,3)}+L_{(1,2,3)} \leq$ $M_{(2,3)}-M_{(1,3,2)}$. (This is because $(1,3,2),(1,2,3) \geq(2,3)$ but $(1,3,2)$ and $(1,2,3)$ are not comparable in the Bruhat order.)

6.3. For $\tau \in \operatorname{Irr} G_{n}$ (not necessarily ladder) let $\mathfrak{m}(\tau)$ denote the multi-segment corresponding to $\tau$. There is an obvious operation of sum of multi-segments. We also have the

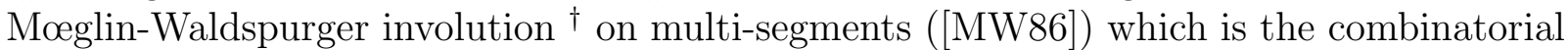
counterpart of the Zelevinsky involution (cf. example 2). Thus, $\mathfrak{m}\left(\tau^{\dagger}\right)=\mathfrak{m}(\tau)^{\dagger}$ for any $\tau$. Suppose that $\tau_{i} \in \operatorname{Irr} G_{n_{i}}, i=1,2$ and that $\pi=\tau_{1} \times \tau_{2}$ is irreducible. Then $\pi^{\dagger}=\tau_{1}^{\dagger} \times \tau_{2}^{\dagger}$ is also irreducible and we have

$$
\left(\mathfrak{m}\left(\tau_{1}\right)+\mathfrak{m}\left(\tau_{2}\right)\right)^{\dagger}=\mathfrak{m}(\pi)^{\dagger}=\mathfrak{m}\left(\pi^{\dagger}\right)=\mathfrak{m}\left(\tau_{1}^{\dagger} \times \tau_{2}^{\dagger}\right)=\mathfrak{m}\left(\tau_{1}^{\dagger}\right)+\mathfrak{m}\left(\tau_{2}^{\dagger}\right)=\mathfrak{m}\left(\tau_{1}\right)^{\dagger}+\mathfrak{m}\left(\tau_{2}\right)^{\dagger} .
$$

However, the condition

$$
\mathfrak{m}\left(\tau_{1}^{\dagger}\right)+\mathfrak{m}\left(\tau_{2}^{\dagger}\right)=\left(\mathfrak{m}\left(\tau_{1}\right)+\mathfrak{m}\left(\tau_{2}\right)\right)^{\dagger}
$$

is not sufficient for the irreducibility of $\tau_{1} \times \tau_{2}$ in general. To wit, let $\tau_{1}=\mathrm{L}(\Delta([2,2]), \Delta([0,1]))$ and $\tau_{2}=\Delta([1,1])$. Then by the algorithm of [MW86] we have $\left(\mathfrak{m}\left(\tau_{1}\right)+\mathfrak{m}\left(\tau_{2}\right)\right)^{\dagger}=$ $\Delta([1,2])+\Delta([1,1])+\Delta([0,0])=\mathfrak{m}\left(\tau_{1}\right)^{\dagger}+\mathfrak{m}\left(\tau_{2}\right)^{\dagger}$. However, $\tau_{1} \times \tau_{2}$ is reducible. (It decomposes as $\mathrm{L}(\Delta([1,2]), \Delta([0,1]))+\mathrm{L}(\Delta([2,2]), \Delta([1,1])) \times \Delta([0,1])$.) 
Needless to say, it would be very interesting to have a combinatorial (or algebraic) description for the irreducibility of parabolic induction in terms of the multisegments of the inducing data. At the moment we are not aware of any such possible characterization (even conjecturally).

6.4. We end up with a conjecture about the general case. Suppose that $\pi=\mathrm{L}\left(\Delta_{1}, \ldots, \Delta_{t}\right)$ and $\Delta_{i}$ does not precede $\Delta_{j}$ for any $i<j$. As before, let $\mathscr{L}$ be the unique maximal submodule of $\Pi:=\Delta_{1} \times \cdots \times \Delta_{t}$ so that $\pi=\Pi / \mathscr{L}$. For any $w \in S_{t}$ let $\Pi_{w}=\Delta_{w^{-1}(1)} \times$ $\cdots \times \Delta_{w^{-1}(t)}$. We will consider the normalized intertwining operators $\mathcal{N}_{w}: \Pi \rightarrow \Pi_{w}$ which are well-defined and non-zero.

For any $i=1, \ldots, t$ let $p_{i}$ be the minimal index $j$ (necessarily bigger than $i$ ) such that $\Delta_{j}$ precedes $\Delta_{i}$. If such $j$ does not exist set $p_{i}=t+1$. (This case will not play a role in what follows.) Similarly, let $q_{i}$ be the maximal index $j$ (necessarily smaller than $i$ ) such that $\Delta_{i}$ precedes $\Delta_{j}$. If $j$ does not exist set $q_{i}=0$. (Again, this case is immaterial.) Let $T$ be the set of pairs $(i, j)$ (necessarily with $i<j$ ) such that $\Delta_{j}$ precedes $\Delta_{i}$ and $p_{i}>q_{j}$. (Note that $p_{i} \leq j$ and $q_{j} \geq i$ if $(i, j) \in T$.) For any $(i, j) \in T$ let $s$ be the transposition $\left(p_{i}, q_{j}\right)$ and let $w$ be the permutation

$$
\begin{aligned}
& 0-0-0-0-0 \quad \Delta_{i} \\
& 0-0=0 \\
& 0 \longleftarrow 0 \\
& w(k)= \begin{cases}k & k<i, \\
p_{i} & k=i, \\
k-1 & i<k \leq q_{j}, \\
k & q_{j}<k<p_{i}, \\
k+1 & p_{i} \leq k<j, \\
q_{j} & k=j, \\
k & k>j .\end{cases} \\
& \circ-\circ-\circ-\circ-\circ-0 \\
& 0-0-0-0-0 \\
& \circ-0-0 \div 0
\end{aligned}
$$

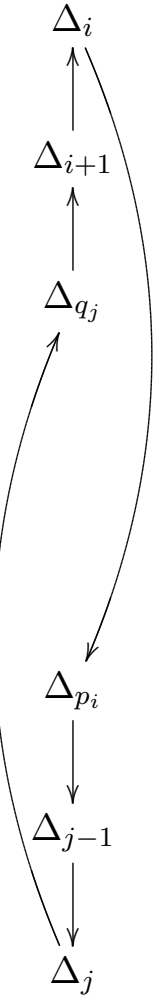

We have $\ell(w)=\ell(s)+\ell(s w)$ and we write correspondingly $\mathcal{N}_{w}=\mathcal{N}_{s}^{\prime} \circ \mathcal{N}_{s w}$ where $\mathcal{N}_{s}^{\prime}: \Pi_{s w} \rightarrow \Pi_{w}$. Since $p_{i}>q_{j}$ the intertwining operator $\mathcal{N}_{s w}$ is an isomorphism: it "moves" $\Delta_{i}$ across $\Delta_{i+1}, \ldots, \Delta_{q_{j}}$ and $\Delta_{j}$ across $\Delta_{j-1}, \ldots, \Delta_{p_{i}}$. The intertwining operator $\mathcal{N}_{s}^{\prime}$ interchanges $\Delta_{i}$ and $\Delta_{j}$ (in their new position). We may decompose $\mathcal{N}_{s}^{\prime}$ into a product 
of rank-one intertwining operators (switching consecutive segments) by choosing a reduced decomposition of $s$. All these operators will be isomorphisms except the one induced from $\Delta_{i} \times \Delta_{j} \rightarrow \Delta_{j} \times \Delta_{i}$. Let $\mathscr{K}_{(i, j)}=\operatorname{Ker} \mathcal{N}_{w}=\mathcal{N}_{s w}^{-1}\left(\operatorname{Ker} \mathcal{N}_{s}^{\prime}\right)$. Finally, set

$$
\mathscr{K}=\sum_{(i, j) \in T} \mathscr{K}_{(i, j)} .
$$

(This is consistent with the $\mathscr{K}$ defined in (4.2) for the case of ladder representations.) Clearly $\mathscr{K} \subseteq \mathscr{L}$.

Conjecture 2. We have $\mathscr{K}=\mathscr{L}$.

This conjecture seems to be the first general conjecture about the fine structure of the Langlands quotient in its standard module. It would have applications, among other things, to questions about existence of functionals with certain invariance properties.

\section{REFERENCES}

[BB05] Anders Björner and Francesco Brenti, Combinatorics of Coxeter groups, Graduate Texts in Mathematics, vol. 231, Springer, New York, 2005. MR MR2133266 (2006d:05001)

[BLM] Alexandru Ioan Badulescu, Erez Lapid, and Alberto Mínguez, Une condition suffisante pour l'irréducibilité d'une induite parabolique de $G L(m, D)$, preprint.

[Bum04] Daniel Bump, Lie groups, Graduate Texts in Mathematics, vol. 225, Springer-Verlag, New York, 2004. MR 2062813 (2005f:22001)

[BZ77] I. N. Bernstein and A. V. Zelevinsky, Induced representations of reductive $\mathfrak{p}$-adic groups. I, Ann. Sci. École Norm. Sup. (4) 10 (1977), no. 4, 441-472. MR 0579172 (58 \#28310)

[CG97] Neil Chriss and Victor Ginzburg, Representation theory and complex geometry, Birkhäuser Boston Inc., Boston, MA, 1997. MR 1433132 (98i:22021)

[CR08] Gaëtan Chenevier and David Renard, Characters of Speh representations and Lewis Caroll identity, Represent. Theory 12 (2008), 447-452. MR MR2465802 (2010f:22014)

[FLO] Brooke Feigon, Erez Lapid, and Omer Offen, On representations distinguished by unitary groups, preprint.

[Hum08] James E. Humphreys, Representations of semisimple Lie algebras in the BGG category $\mathscr{O}$, Graduate Studies in Mathematics, vol. 94, American Mathematical Society, Providence, RI, 2008. MR 2428237 (2009f:17013)

[LS90] V. Lakshmibai and B. Sandhya, Criterion for smoothness of Schubert varieties in $\mathrm{Sl}(n) / B$, Proc. Indian Acad. Sci. Math. Sci. 100 (1990), no. 1, 45-52. MR 1051089 (91c:14061)

[MW86] C. Mœglin and J.-L. Waldspurger, Sur l'involution de Zelevinski, J. Reine Angew. Math. 372 (1986), 136-177. MR 863522 (88c:22019)

[Rod82] François Rodier, Représentations de $\mathrm{GL}(n, k)$ où $k$ est un corps p-adique, Bourbaki Seminar, Vol. 1981/1982, Astérisque, vol. 92, Soc. Math. France, Paris, 1982, pp. 201-218. MR 689531 (84h:22040)

[Sta97] Richard P. Stanley, Enumerative combinatorics. Vol. 1, Cambridge Studies in Advanced Mathematics, vol. 49, Cambridge University Press, Cambridge, 1997, With a foreword by Gian-Carlo Rota, Corrected reprint of the 1986 original. MR MR1442260 (98a:05001)

[Sta99] _ Enumerative combinatorics. Vol. 2, Cambridge Studies in Advanced Mathematics, vol. 62, Cambridge University Press, Cambridge, 1999, With a foreword by Gian-Carlo Rota and appendix 1 by Sergey Fomin. MR 1676282 (2000k:05026) 
[Tad86] Marko Tadić, Classification of unitary representations in irreducible representations of general linear group (non-Archimedean case), Ann. Sci. École Norm. Sup. (4) 19 (1986), no. 3, 335-382. MR 870688 (88b:22021)

[Tad87] _ Unitary representations of $\mathrm{GL}(n)$, derivatives in the non-Archimedean case, V. Mathematikertreffen Zagreb-Graz (Mariatrost/Graz, 1986), Ber. Math.-Statist. Sekt. Forschungsgesellsch. Joanneum, vol. 274, Forschungszentrum Graz, Graz, 1987, pp. Ber. No. 281, 19. MR 904061 (89c:22028)

[Tad95] M. Tadić, On characters of irreducible unitary representations of general linear groups, Abh. Math. Sem. Univ. Hamburg 65 (1995), 341-363. MR 1359141 (96m:22039)

[Tad06] Marko Tadić, Representation theory of $\mathrm{GL}(n)$ over a p-adic division algebra and unitarity in the Jacquet-Langlands correspondence, Pacific J. Math. 223 (2006), no. 1, 167-200. MR 2221023 (2007b:22020)

[Zel80] A. V. Zelevinsky, Induced representations of reductive $\mathfrak{p}$-adic groups. II. On irreducible representations of $\operatorname{GL}(n)$, Ann. Sci. École Norm. Sup. (4) 13 (1980), no. 2, 165-210. MR MR584084 (83g:22012)

[Zel81] A. V. Zelevinskiŭ, The p-adic analogue of the Kazhdan-Lusztig conjecture, Funktsional. Anal. i Prilozhen. 15 (1981), no. 2, 9-21, 96. MR MR617466 (84g:22039)

[Zel85] _ Two remarks on graded nilpotent classes, Uspekhi Mat. Nauk 40 (1985), no. 1(241), 199-200. MR MR783619 (86e:14027)

Institute of Mathematics, Hebrew University of Jerusalem, Jerusalem 91904, IsRael E-mail address: erezla@math.huji.ac.il

Institut de Mathématiques de Jussieu, Université Paris 6. 4, Place Jussieu. 75005 Paris, FRANCE. URL: http://www.institut.math.jussieu.fr/ minguez/

E-mail address: minguez@math.jussieu.fr 